DOF/ER/14 207--2

DE93 008296

\title{
PARTICIPATION IN THE CREEDE SCIENTIFIC DRILLING PROJECT AS ON-8ITE PRINCIPAL INVESTIGATOR
}

\author{
Final Report \\ Grant No. DE-FG02-91ER14207 \\ Jeffrey B. Hulen \\ University of Utah Research Institute \\ 391-C Chipeta Way \\ Salt Lake City, UT 84108
}

June 1992

\section{DISCLAIMER}

This report was prepared as an account of work sponsored by an agency of the Unitod States Government. Neither the United States Government nor any agency thereof, nor any of their employees, makes any warranty, express or implied, or assumes any legal liability or responsibility for the accuracy, completeness, or usefulness of any information, apparatus, pruduct, or process disclosed, or represents that its use would not infringe privately owned rights. Reference herein to any specific commercial product, process, or service by trade name, trademark, manufacturer, or otherwise does not necessarily constitute or imply its endorsement, recommendation, or favoring by tine United States Government or any agency thereof. The views and opinions of authors expressed herein do not necessarily state or reflect those of the United States Government or any agency thereof. 


\section{PARTICIPATION IN THE CREEDE SCIENTIFIC DRILLING PROJECT AS ON-SITE PRINCIPAL INVESTIGATOR}

Late in 1991, the U.S. Geological survey (USGS), in partnership with the National Science Foundation (NSF) and the Department of Energy (DOE) completed two intermediate-depth coreholes in the moat of the $26 \mathrm{~m} . \mathrm{y}$-old creede caldera in southern Colorado (Fig. 1) as part of the U.S. Base Program of continental Scientific Drilling. Chief Scientist for the project was (and remains) Philip M. Bethke (USSG, Reston, VAl: Thomas H. Moses, Jr. (USGS, Menlo Park, CA) served as Chief Engineer. Jeffrey B. Hulen, University of Utah Research Institute (UURI), was funded by the DOE's office of Basic Energy Sciences to work closely with these key individuals in the role of on-site Principal Investigator (P.I.); Wayne Campbell (USGS, Reston) was assistant on-site P.I. Tonto Drilling Services of Salt Lake City, Utah (Wayne Beaupre, Supervisor) completed the actual coring. Six additional drill-site geoscientists (F. Gay, N. Foley, D. Hayba, A. Hopkins, D. Larsen, and R. Streufert) were essential for core lngging and curation, and undertook additional responsibilities to be elaborated later in this report. This on-site management team was responsible for ensuring that the project's scientific and technical objectives were smoothly and successfully achieved. Cores obtained from the project are currently under detailed investigation by 23 Principal Investigators representing 12 U.S. institutions. Additional investigators with ideas for further work on the creede cores are encouraged to participate, and should contact Chief scientist Philip Bethke at the USGS in Reston, VA. 


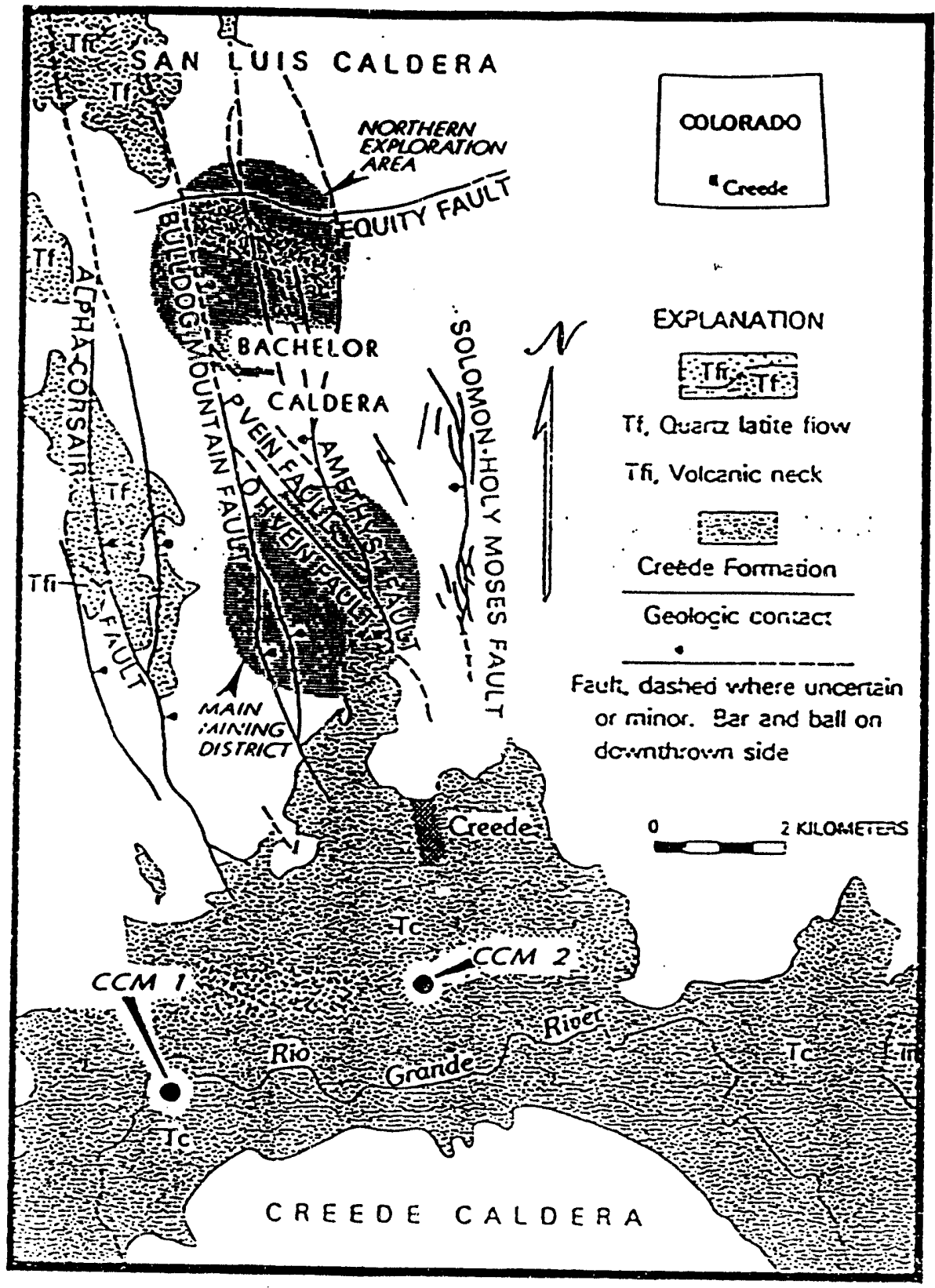

FIGURE 1. LOCATION MAP. CREEDE SCIENTIFIC DRILLING PROJECT 
Scientific questions addressed by the creede scientific Drilling Project were as follows (Bethke et al., 1992): (1) Did the lacustrine sedimentary sequence filling the moat of creede caldera serve as reservoir for the moderately-saline aqueous fluids which scavenged and then transported silver and base metals to oredepositional sites for the rich epithermal deposits of the creede mining district (Fig. 1)?; (2) what were the chemical and isotopic compositions of these fluids prior to their entry into the creede fracture (later vein) system; (3) how did these chemical and isotopic compositions evolve in transit to the ore-depositional site?; (4) how did the creede caldera form and evolve?; (5) what is the present thermal regime in Creede caldera moat? ...the paleothermal regime?; (5) what are the hydrologic transport properties of the moat sedimentary rocks?; (6) what diagenetic or hydrothermal veins disrupt the moat sedimentary sequence, and what do their paragenetic relationships, mineralogic compositions, fluid-inclusion characteristics, and stable-isotope systematics reveal about evolution of the creede hydrothermal system?

Two Creede caldera moat drill holes were completed for this project. The deepest $(725 \mathrm{~m}), \mathrm{CCM}-2$ or Airport $1-6$ (Fig. 1) was completed in the central portion of the moat along strike with the Creede district epithermal vein complex, partially on the premise that fractures extending southward from what was to become the district would have guided moat fluids as they entered the Creede hydrothermal system. A second hole (CCM-1, or Hosselkus 1-10; Fig. 2) was drilled to a depth of $375 \mathrm{~m}$ about $4 \mathrm{~km}$ to the west, well away from major paleohydrothermal fluid flow and hopefully free of 
hydrothermal overprints so as to provide background material to compare with possibly altered core from CCM-2.

The two holes were drilled by Tonto Drilling services, working 24 hours per day in two 12 -hour shifts. The Tonto Drilling team included veterans of past scientific and geothermal drilling projects such as Jeff Riley, Justin Semadeni, and Jerry Gillespie. Under the direction of Wayne Beaupre, Tonto obtained better than 998 core recovery for both the Creede holes; individual cores. reaching more than $10 \mathrm{ft}$ in length were frequently retrieved, $\cdots$ assuring a wealth of solid sample for concerned Principal Investigators.

An on-site science team consisting of the individuals listed above complemented Tonto's superb efforts by providing round-theclock core logging, fluid sampling, and drill-site monitoring and supervision. The team worked in shifts staggered slightly from Tonto's schedule. The principal job of the on-site science team was careful curation, labeling, boxing, and logging of the cores after provision of same by the Tonto drilling crew. Depending on drilling rate, this task required either a two-person or threeperson team -- the latter particularly when drilling was troublefree, the rocks were competent but soft, and drilling rates exceeded $150 \mathrm{ft}$ per day.

Upon reception at the rig from Tonto personnel, each core was carefully washed to remove drilling fluids, dried (with a portable, hand-held, forced-air heater), marked, and oriented, so that should an unlikely accident occur, the individual pieces could be readily re-assembled. The cores were then boxed, and logged in detail at 
a scale of $1^{\prime \prime}=1 \mathrm{ft}$, with attention to lithology, fracturing, mineralization, organic-matter content, alteration, and sedimentary structures. Prior to the project, at the suggestion of computer expert Wayne Campbell, a suite of representative rock samples from Creede caldera and vicinity were assigned standard numbers, so that their counterparts in core could be categorized quickly and efficiently for subsequent computer-assisted illustration. These logs were abstracted to prepare the summary logs shown in Appendix 2 .

Additional on-site activities pertaining to core included collection of special samples for certain P.I.'s as well as laboratory natural gamma and magnetic-susceptibility logging for provision of ground truth to aid in interpretation of actual downhole gamma and magnetic susceptibility logs. Special samples included those tightly foil-wrapped and waxed to preserve natural formation fluids, and those containing particularly large amounts of organic debris. The above-ground geophysical logging of the core was carried out as time permitted; rapidly-retrieved core required full-time attention to the more important geologic logging described above. In support of detailed studies to be carried out later by the broader creede scientific consortium, samples of all drilling fluids and additives were systematically collected and retrieved -- for example, each time a new load of drilling water was obtained from the Creede culinary water supply. Actual water samples were also retrieved from several moat aquifers for those carrying out hydrogeochemical studies in support of the project. As an interesting aside, a high-level aquifer in Rio Grande gravel 
deposits in $\mathrm{CCM}-2$ was discovered to produce copious quantities of water which the town of Creede may use to supplement its existing culinary water supplies.

Besides carrying out the duties specified above, the on-site science team arranged and monitored site visits by scientific and other parties, and kept the town of creede apprised of new developments in the project (such as the discovery of potential culinary water in $\mathrm{CCM}-2)$. Prior to drilling, we conducted a town meeting during which the project's objectives, schedule, etc. were presented to the attendees. "Open-houses" were also held at the drill site so that people from creede could observe drilling and related scientific activities in progress and ask questions of the on-site scientific, engineering, and drilling staff.

The two holes have already provided a wealth of new information about the creede caldera moat and fluids residing in it at the time of formation. Through detailed studies of the core and fluids retrieved during this project, in combination with geophysical logging and other allied projects, Principal Investigators are beginning to uncover hitherto unknown facets of the Creede district's tectonic, volcanic, and hydrothermal histories.

Both Creede moat coreholes penetrated principally the moatfilling sedimentary sequence of the Creede caldera (Appendix 1) -the lacustrine facies of the creede Formation. $\mathrm{CCM}^{-1}$ (Fig. 1) drilled through $375 \mathrm{~m}$ of creede Formation and $55 \mathrm{~m}$ of caldera-wall landslide breccias. CCM-2 (Fig. 2) penetrated $500 \mathrm{~m}$ of Creede Formation and $225 \mathrm{~m}$ of breccias, zeolitized tuffs, and 
miscellaneous epiclastic debris. In both holes, the creede Formation comprises principally finely-laminated to thinly bedded, zeolitized tuffaceous siltstone and sandstone probably deposited by sublacustrine turbidites. Locally interrupting this sequence are debris-flow breccias up to $15 \mathrm{~m}$ thick, possible subaqeous felsic pyroclastic flow rocks, and subaqueous felsic fallout tuffs. The Creede Formation in the upper third of each hole hosts conspicuous, rice-grain-shaped calcite pseudomorphs after the cold-waterprecipitated hydrous calcium carbonate ikaite. Disseminated pyrite in minute quantities is ubiquitous in the creede in both holes; and a few laminae are dominated by this sulfide. In the snowshoe Mountain ignimbrite intersected in the lower portion of CCM-2 (Appendix I), calcium carbonate-pyrite-clay veins are common; a few of these are prominently open and host well-developed pyrite stalactites enveloped by bladed calcite. These and other critical features of the core as observed on-site during the drilling project are recorded in the summary logs of Appendix 1.

\section{REFERENCES}

Bethke, P.M., Lipman, P.W., Barton, P.B., Jr., Daniels, J.J., Foley, N.K., and Hayba, D.O., 1987, Research drilling in the mineralized hydrothermal system at creede, Colorado: Research Proposal Submitted to DOSECC, Inc., 129 p.

Bethke, P.M., Hulen, J.B., Moses, T.H., Jr., and Campbell, W.R., 1992, Research drilling the moat of the $26 \mathrm{Ma}$ Creede caldera, San Juan Mountains, SW Colorado, USA (abs.): Paris, Int. Symp. on Continental Sci. Drilling.

Hulen, J.B., 1991, Proposal for participation in the creede Scientific Drilling Project as on-site principal Investigator: Proposal to U.S. Dept. of Energy, Office of Basic Energy Sciences, $17 \mathrm{p}$. 
Creede Scientific Drilling Project

(USGS - NSF - DOE)

\title{
Field Geologic Log Summaries ( 1 in. $=12 \mathrm{ft}$.) \\ for Coreholes CCM-2 (Airport 1-6) and CCM-1 (Hosselkus 1-10)
}

\author{
Jeffrey B. Hulen \\ University of Utah Research Inscirute
}

prepared from 1 in. $=1.5 \mathrm{ft}$-scale on-site geologic logs by

P.M. Bethke

W.R. Campbell

N. Foley

F.E. Gay

D.O. Hayba

A.J. Hopkins

J.B. Hulen

D. Larsen

R. Streufert 


\section{Explanation for Sedimentary Structure Symbols}

1. Calcite pseudomorphs after ikaite(?); so-called "rice grain" structures

m Convolute stratification

1. ... Normally graded bedding (arrow shows

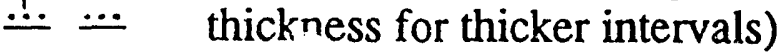

$\ldots \quad$ Reverse graded bedding

$\because \quad$ : Symmetrically graded bedding

$\mho \tau^{\mho}$ Load structures

$\mathscr{C}$ Flame structures

$\perp \quad$ Injection structures

ค Algal mounds (?)

^ิ Unidirectional ripples

$f$ Penecontemporaneous fault

Rip-up clast

- Calcareous concretion

$\square \triangleright \quad$ Autoclast

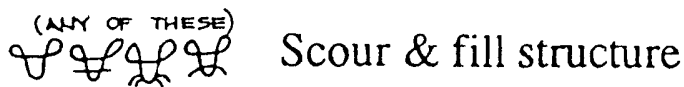

4 Cross-bedding

B Burrow(?)

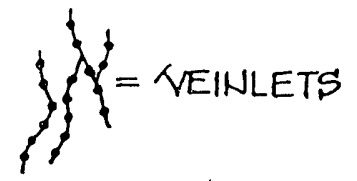

$\varnothing \varnothing \quad$ Plant fossil

vo Mudcracks

Acknowledgement: Funding for Hulen's participation in the Creede Scientific Drilling Project was provided by the U.S. Department of Energy, Office of Basic Energy Sciences, Grant No. DE-FG02-91ER 14207 


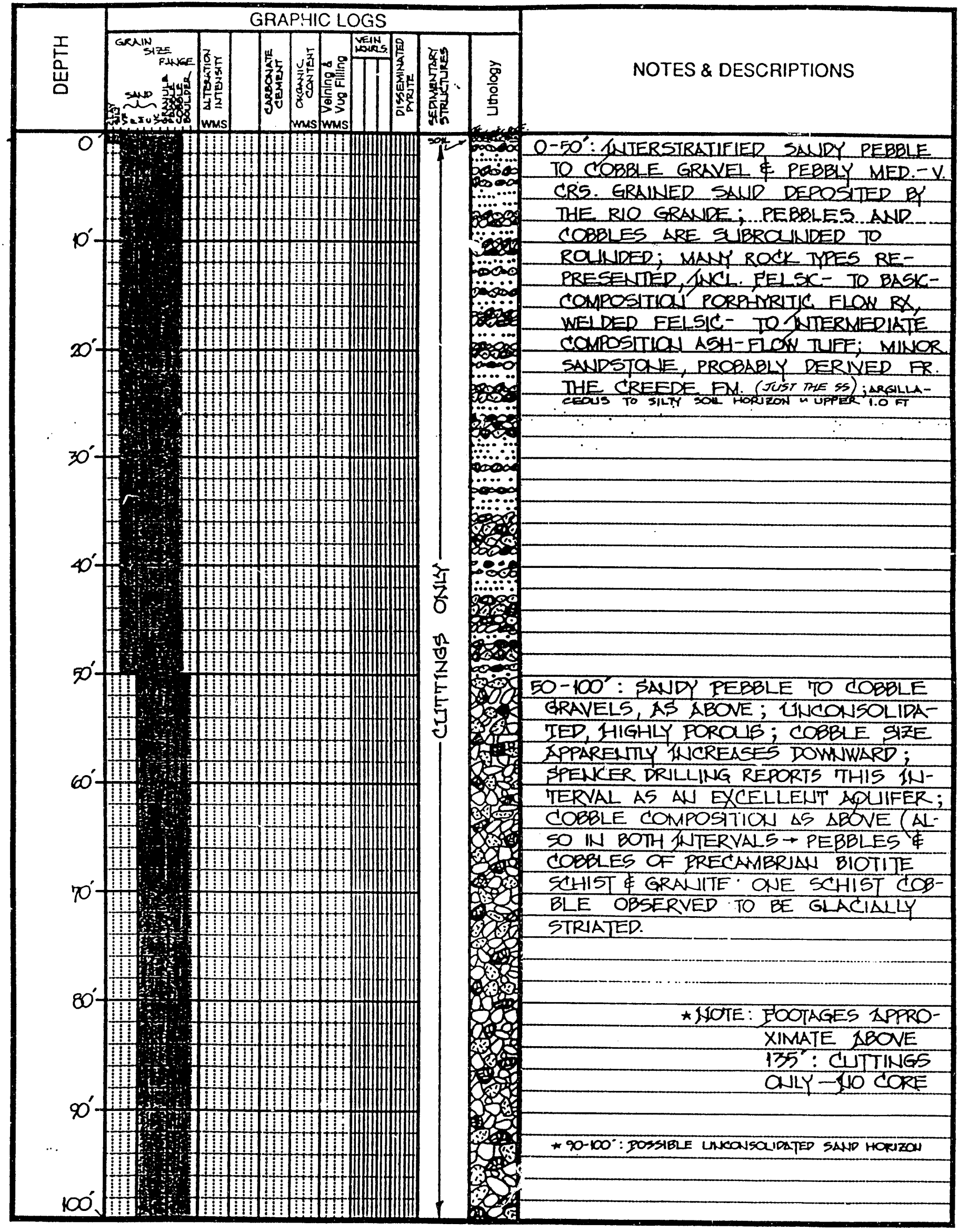

CREEDE CALDERA

MOAT DRILI HOLE NO. 2
SUMMARY

CORE LOG
Logged by HUF HUEN

Date OCTOBER 24-26, 1991 


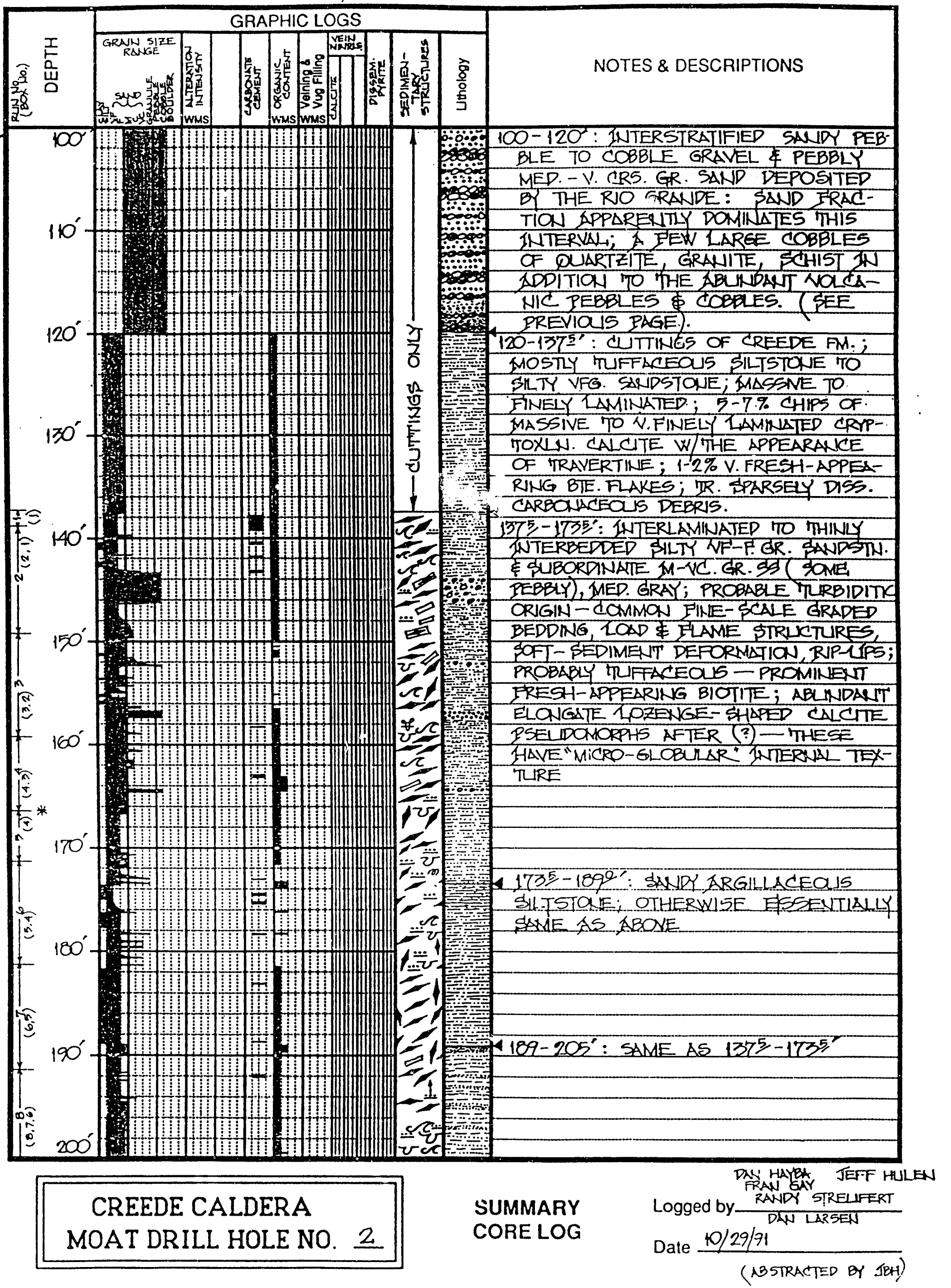




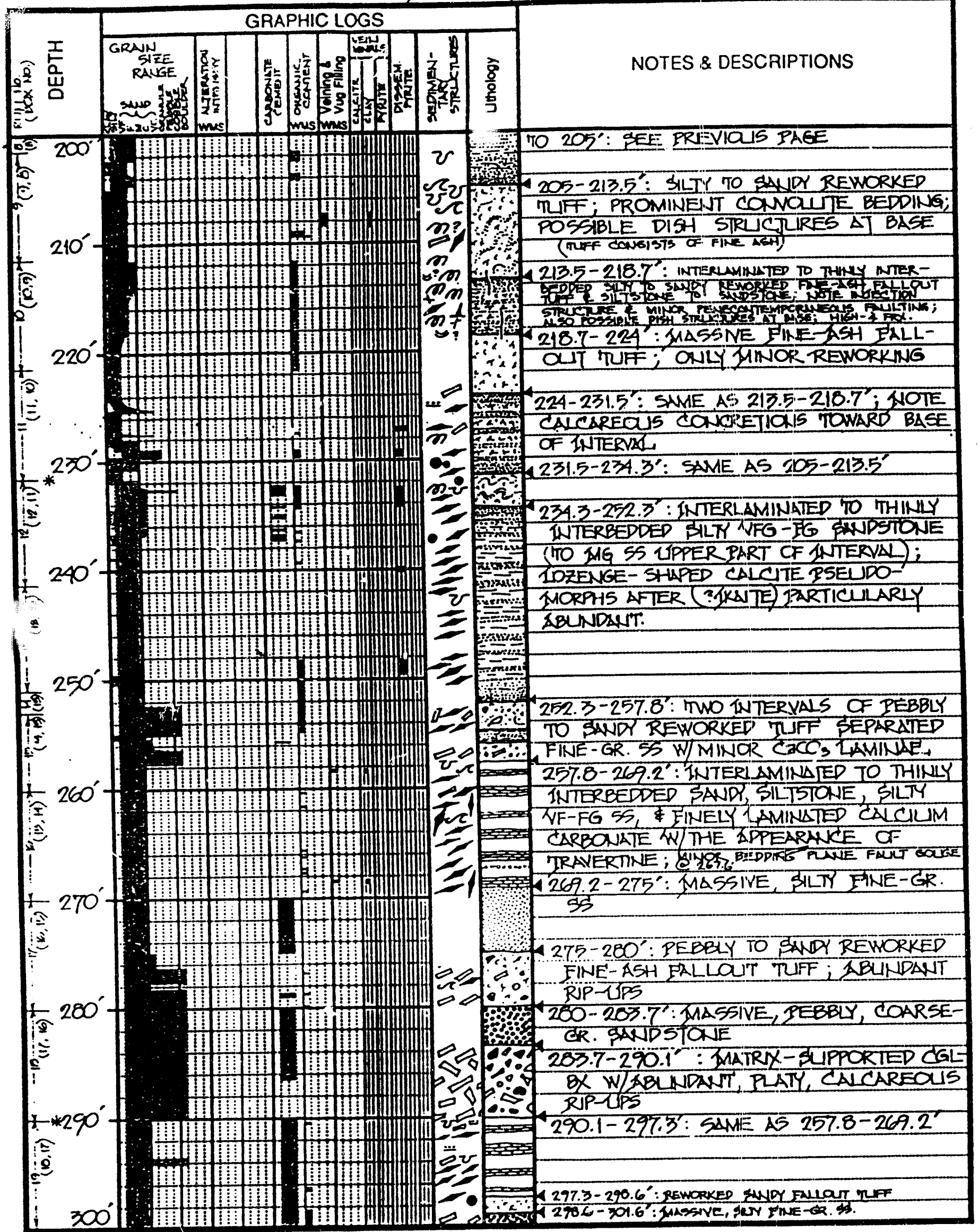

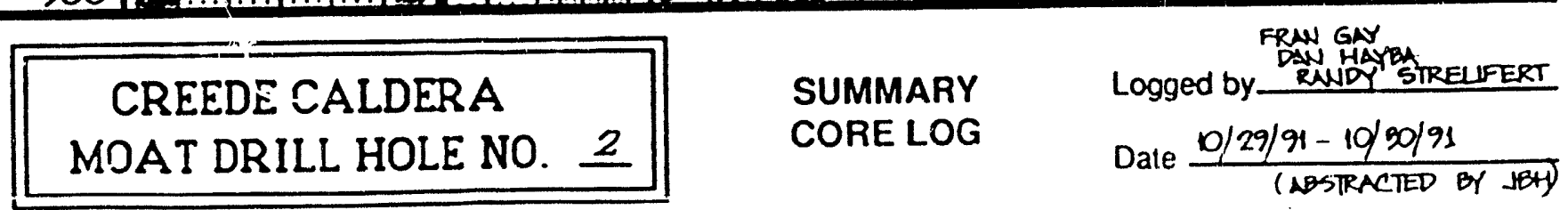




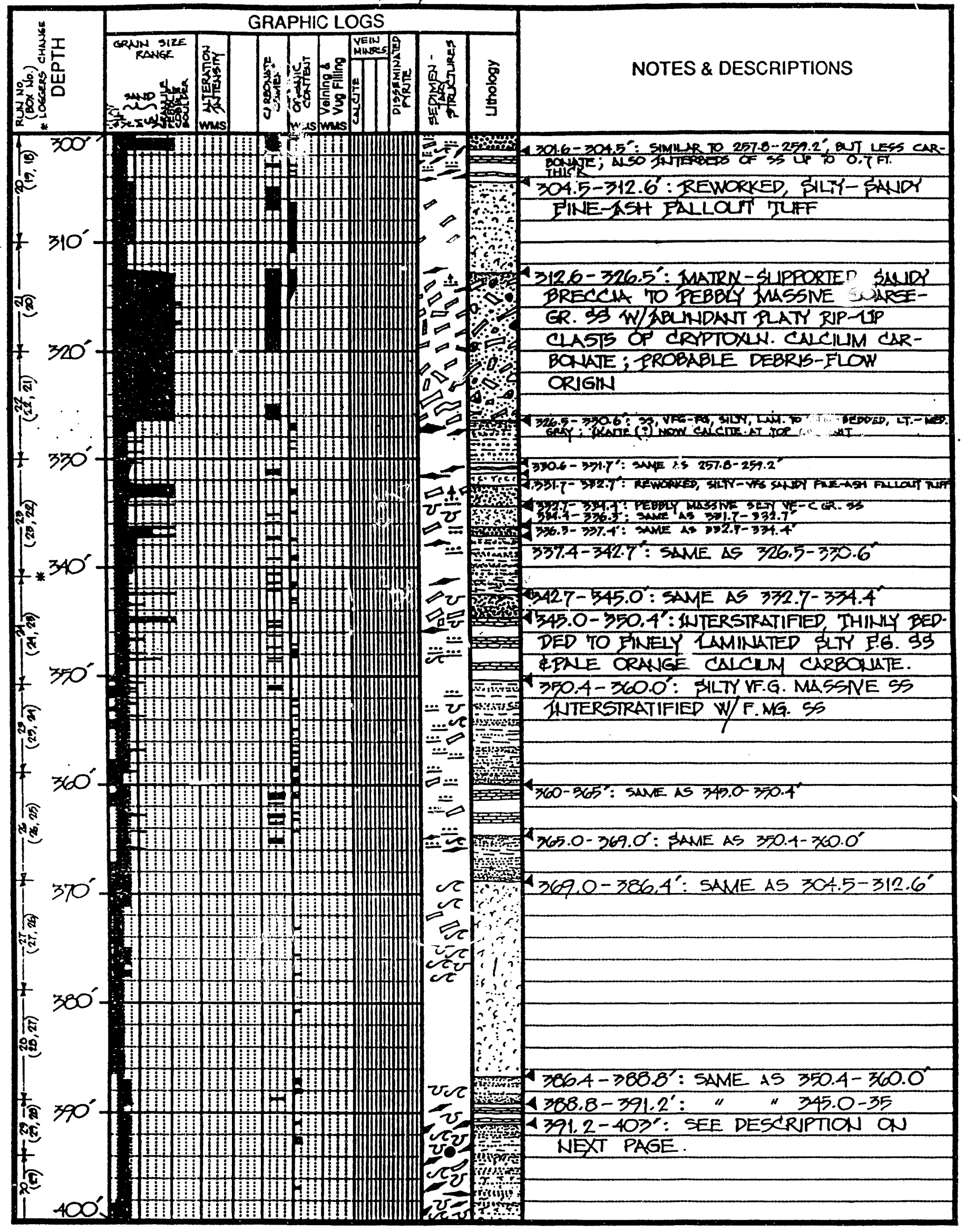

CREEDE CALDERA MOAT DRILL HOLE NO. $\underline{2}$
SUMMARY

CORE LOG
Logged by DAN LARSEN, FRAN GAY

Date NOV. 2, 1991 (DSTRATED OY SEY) 


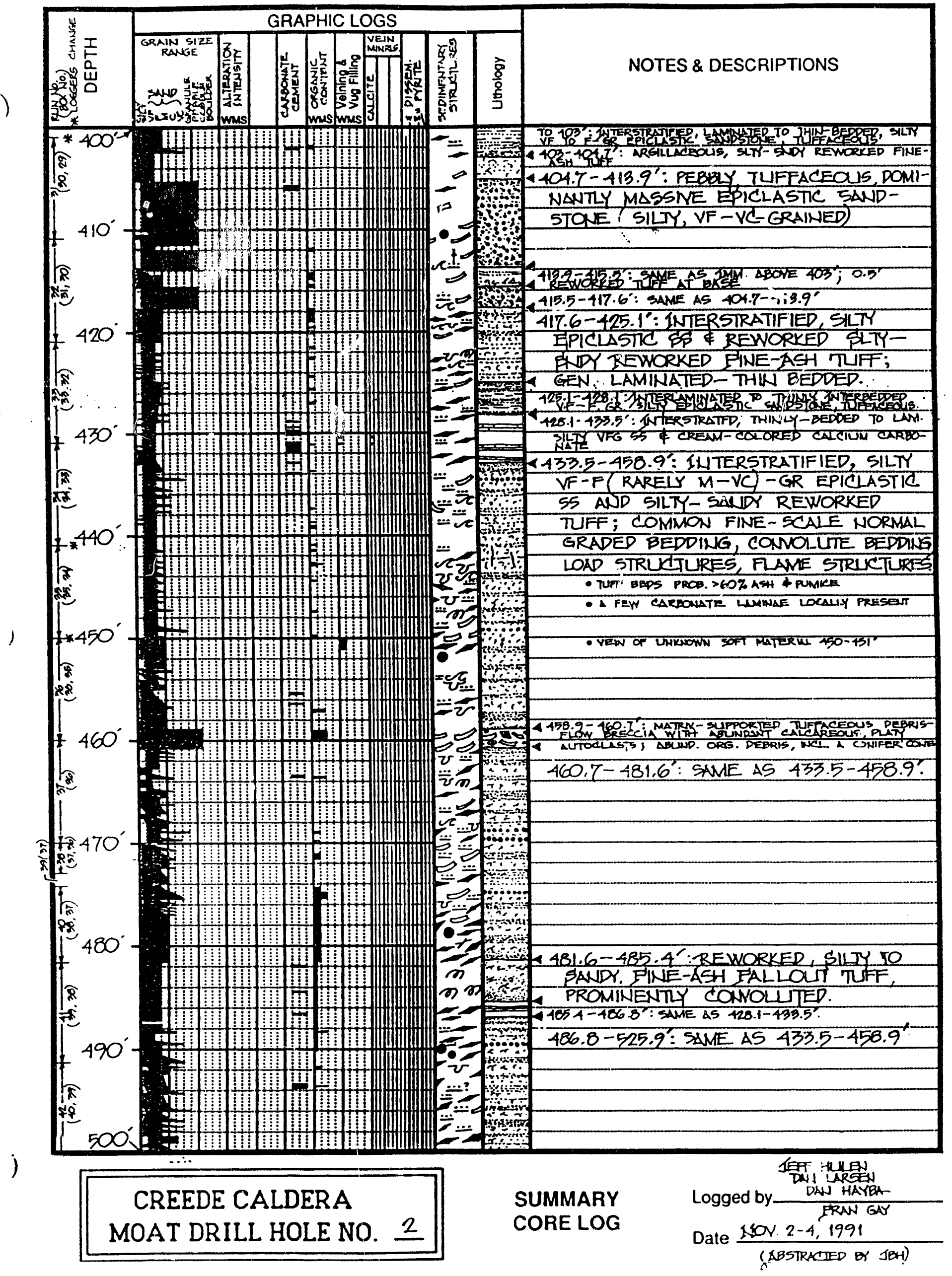




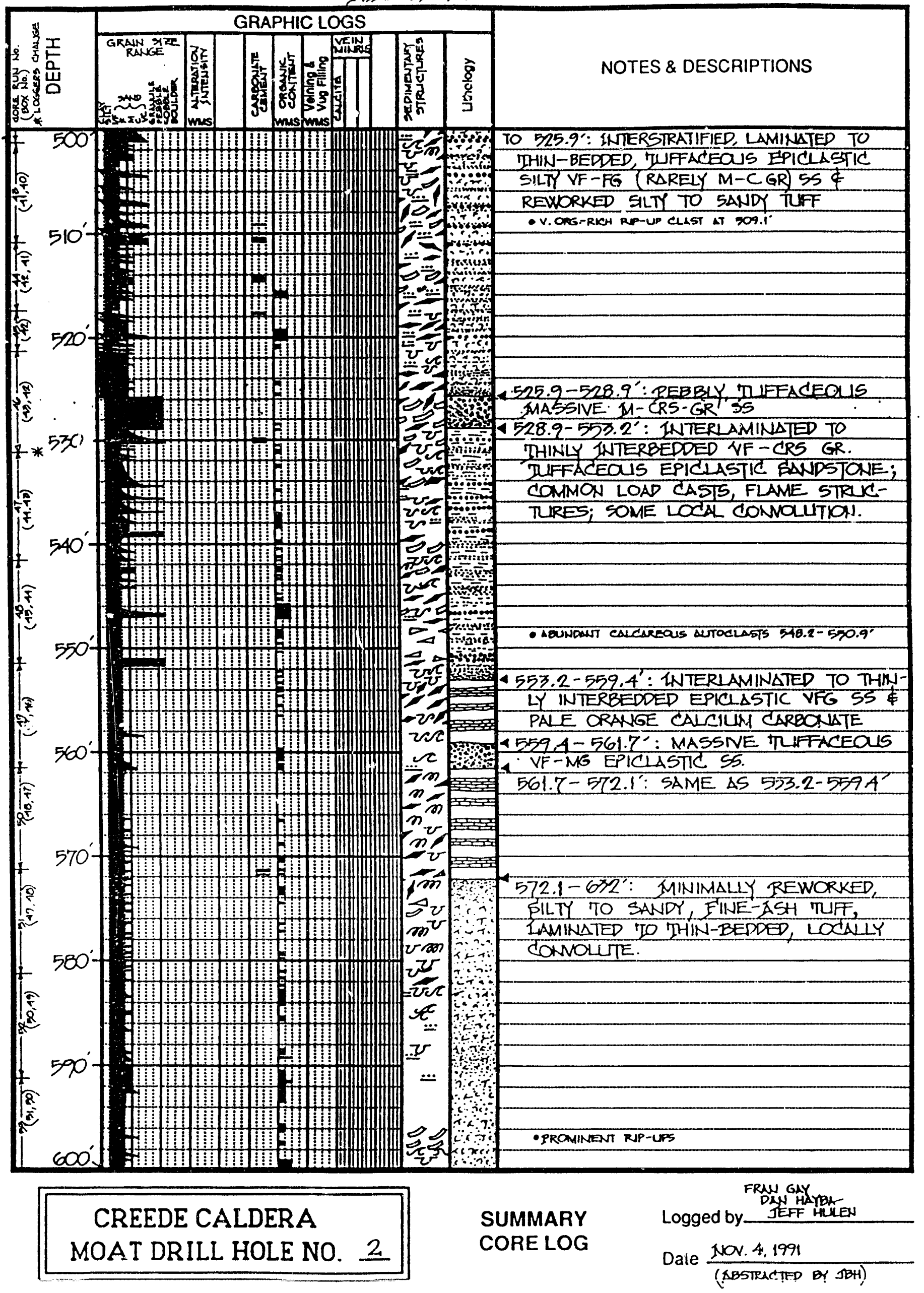




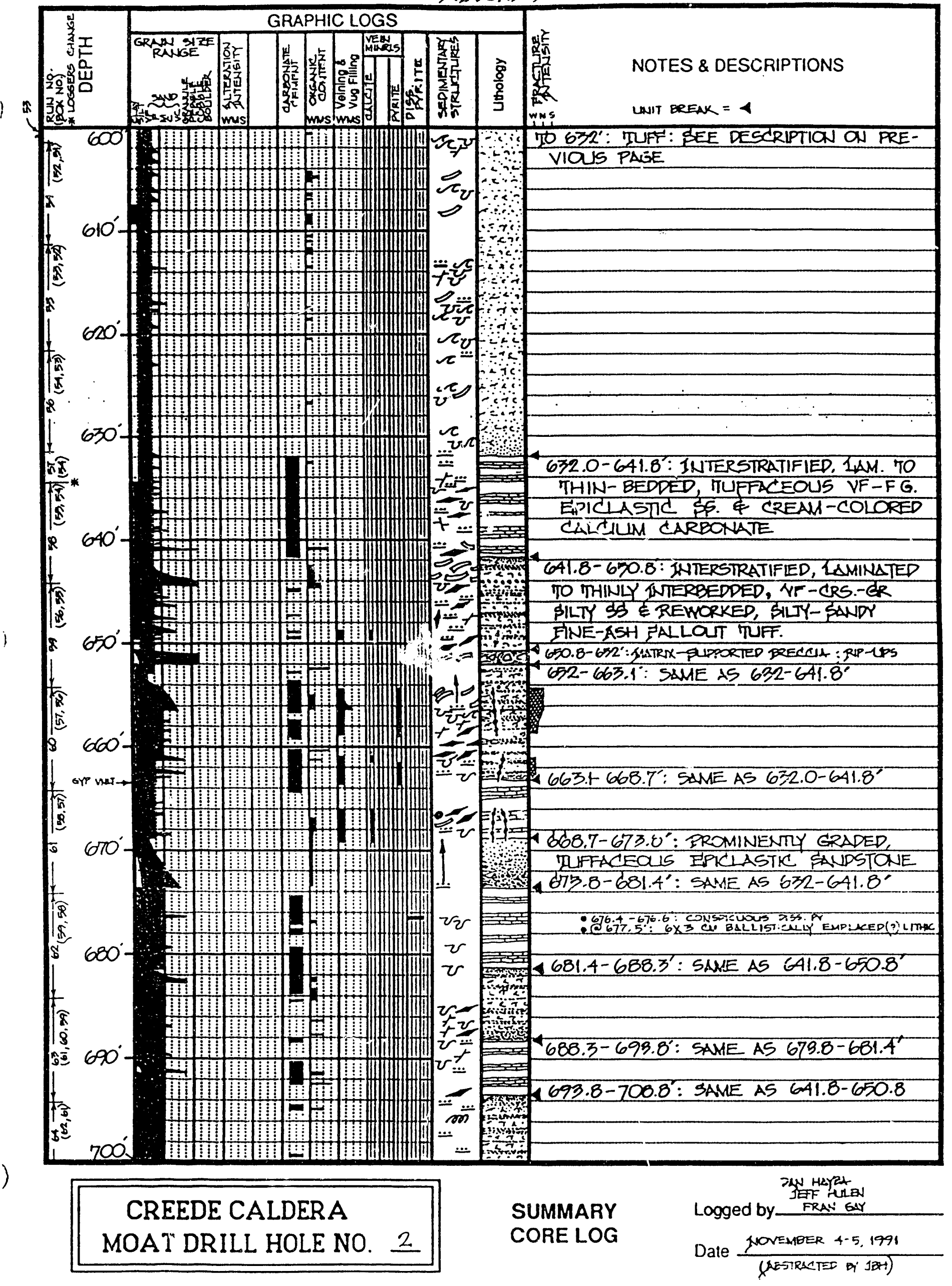




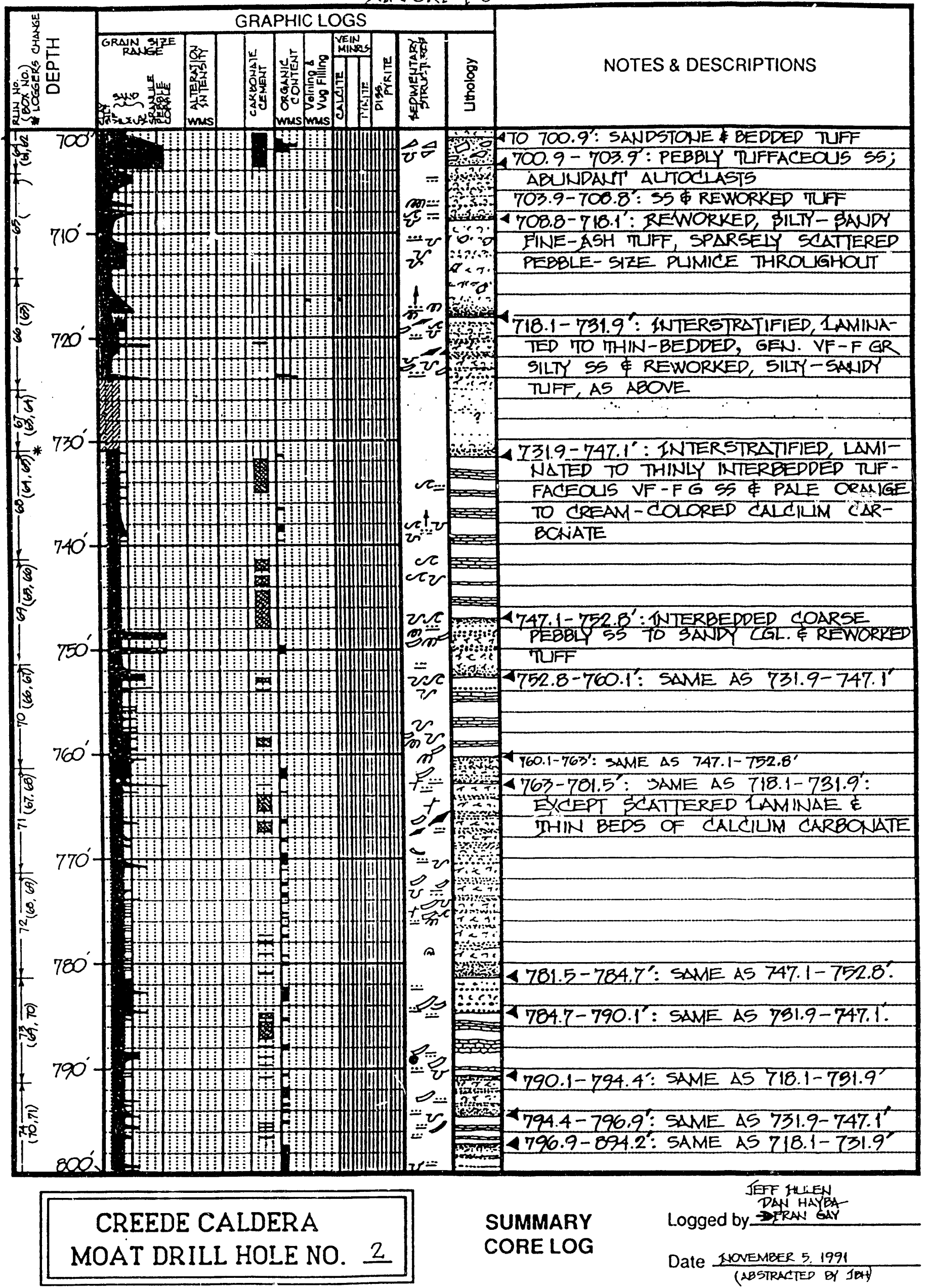




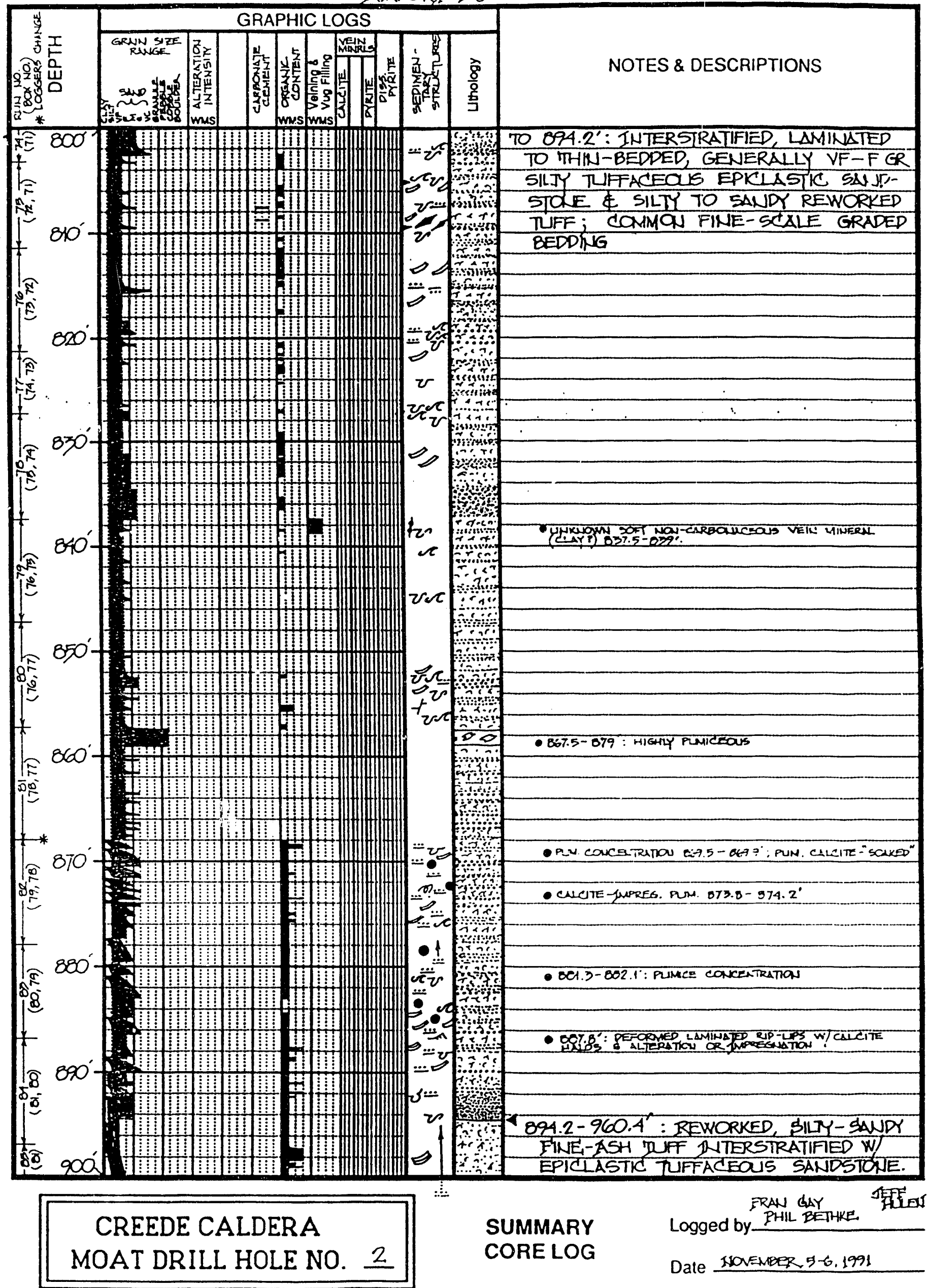




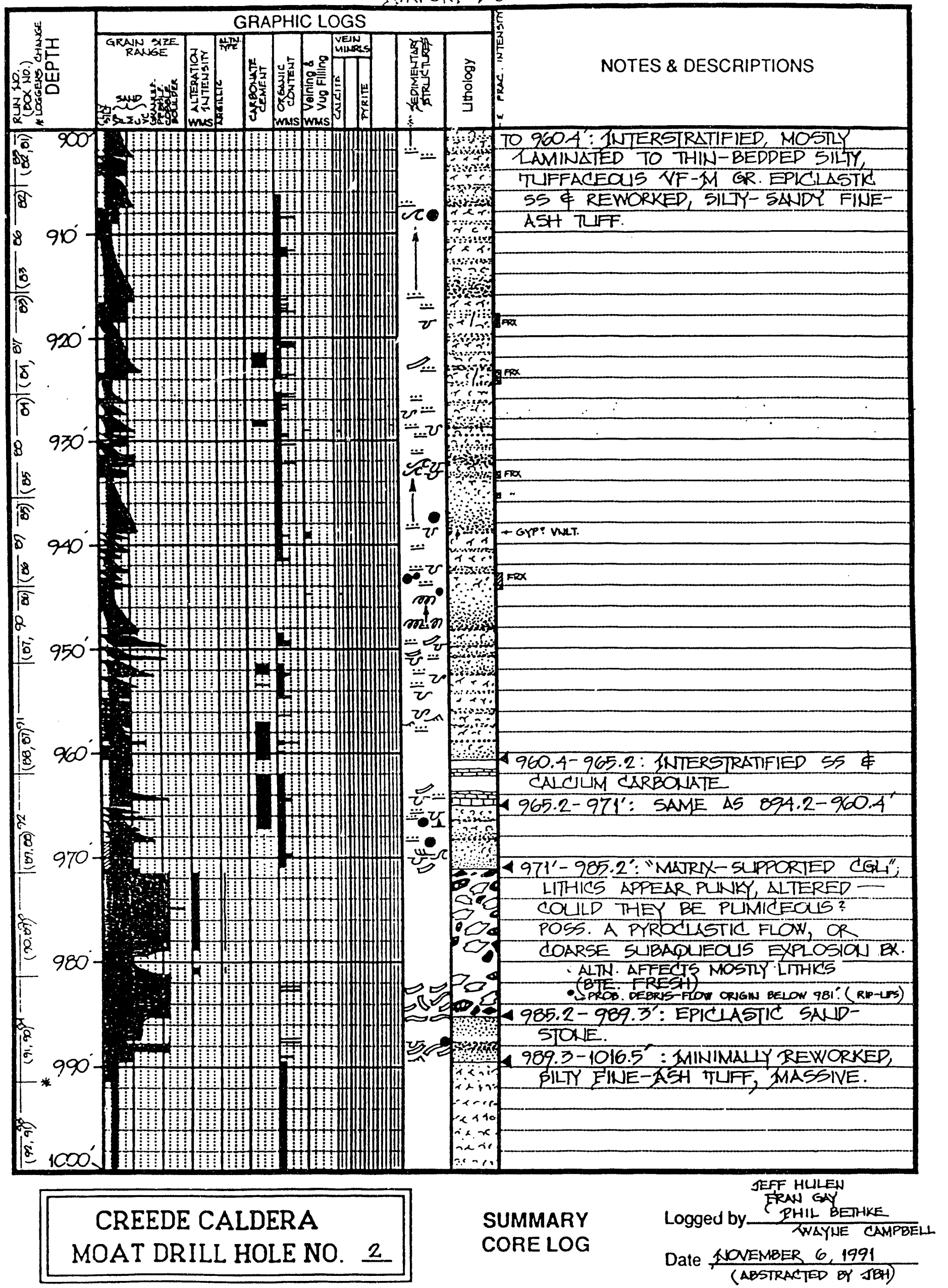



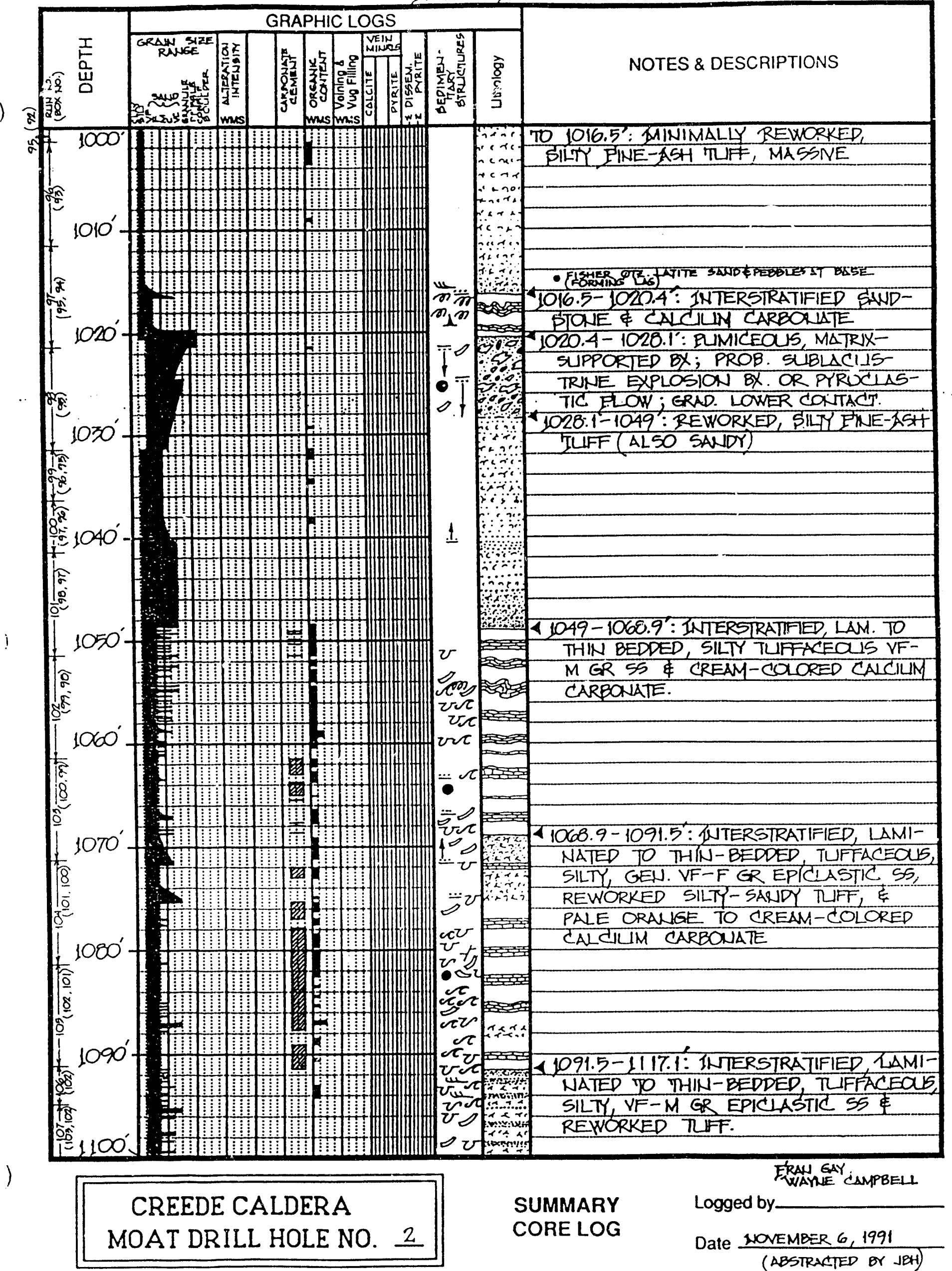

SUMMARY CORE LOG
FRAN GAY CAMPBELLL Logged by

Date NOVEMBER 6, 1991 (ABSTRATTED BY JEH) 


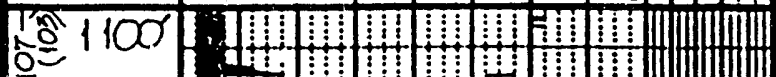

은

*

成

으 $1110^{\prime}$

10

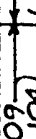

造 1120

$\underline{\underline{0}}$

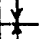

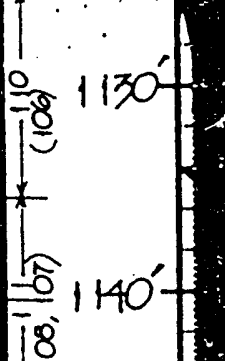

10

81150

To

$\underline{2}$

$\sqrt{\sigma} 1160^{\prime}$

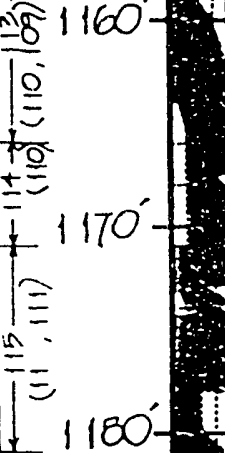

gas

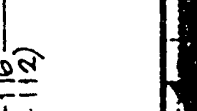

$\equiv 1190^{\prime}$

管

$\stackrel{n}{=}$

$\stackrel{\square}{\Xi} 1200$

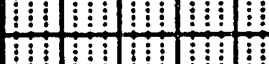

(1)

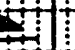

4

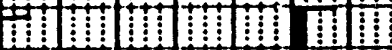

$+1+1$

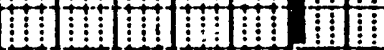

$2+1+11$

II 1 :

$+11+1$

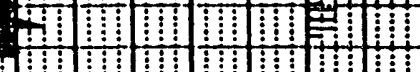

$11: 11$

$1: 19: 10$

$+1 \div$

!1:!I!:

$1+1: 1:$

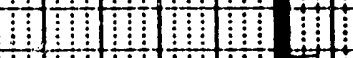

$+1$

(1) $1:+1$

$4 \div$

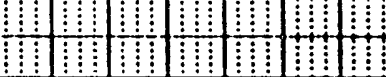

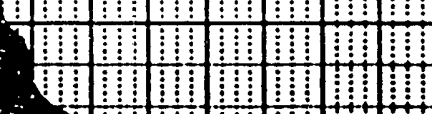

and

10

-

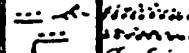

I.

\section{In}

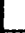

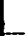

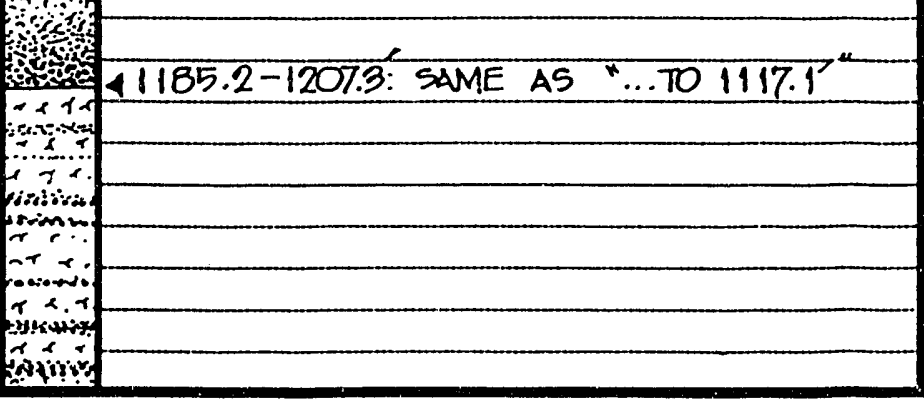

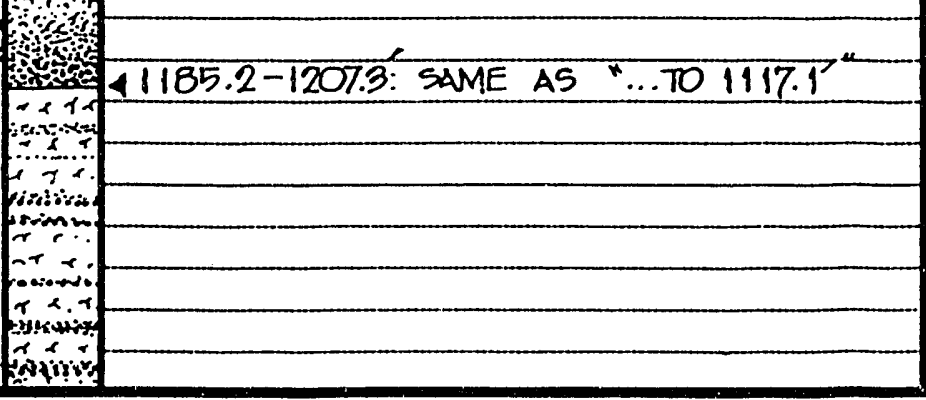

$1131.7-11.42 .5^{\circ}$ SMME AS.1091.5-1117.1 INTERBEDDED SILTY VF-F GR S5, SNDY SLTS, \& SUBORDINATE SILTY CLAY-SIZE MIATERIAL IN MED. BRONN BLNDS WHICH ARE PROBABLY REWORKED ASH; PROBABLE TURBIDITTC ORIGIN.

41117.1-i131.7: REWORKED, SILTY TO SANDY, EINE-ASH FALLON TUFF GRNN SIZE COARSEUING DOWNANADD

$1142.3-1166:$ MINIMALLY REXORKED, FINE-ASH FALLOUT TLFF LT. -MED. GREENISH-GRAY, COARSENING DOWHWARD; PROMINIENTY CONVOWTED; OQVIOUS DISSEMINATED PYRTE.

- $1160.6-1161.2$ LARGE, AMOBOID, CALCAREOUS 1166-1178: SAME AS "..TO 1117.'! .

$1178-1185.2$ : SAME AS $1117.1-1131.7^{\prime}$

CREEDE CALDERA MOAT DRILL HOLE NO. $\underline{2}$ CORE LOG
JEF HUN Logged by $\frac{\text { ERAN GAY }}{\text { WWYYE CAMPBEIL }}$

Date NOYEMBER 6-7, 1992 ( $\triangle B S T R A C T E D$ BY SBH) 


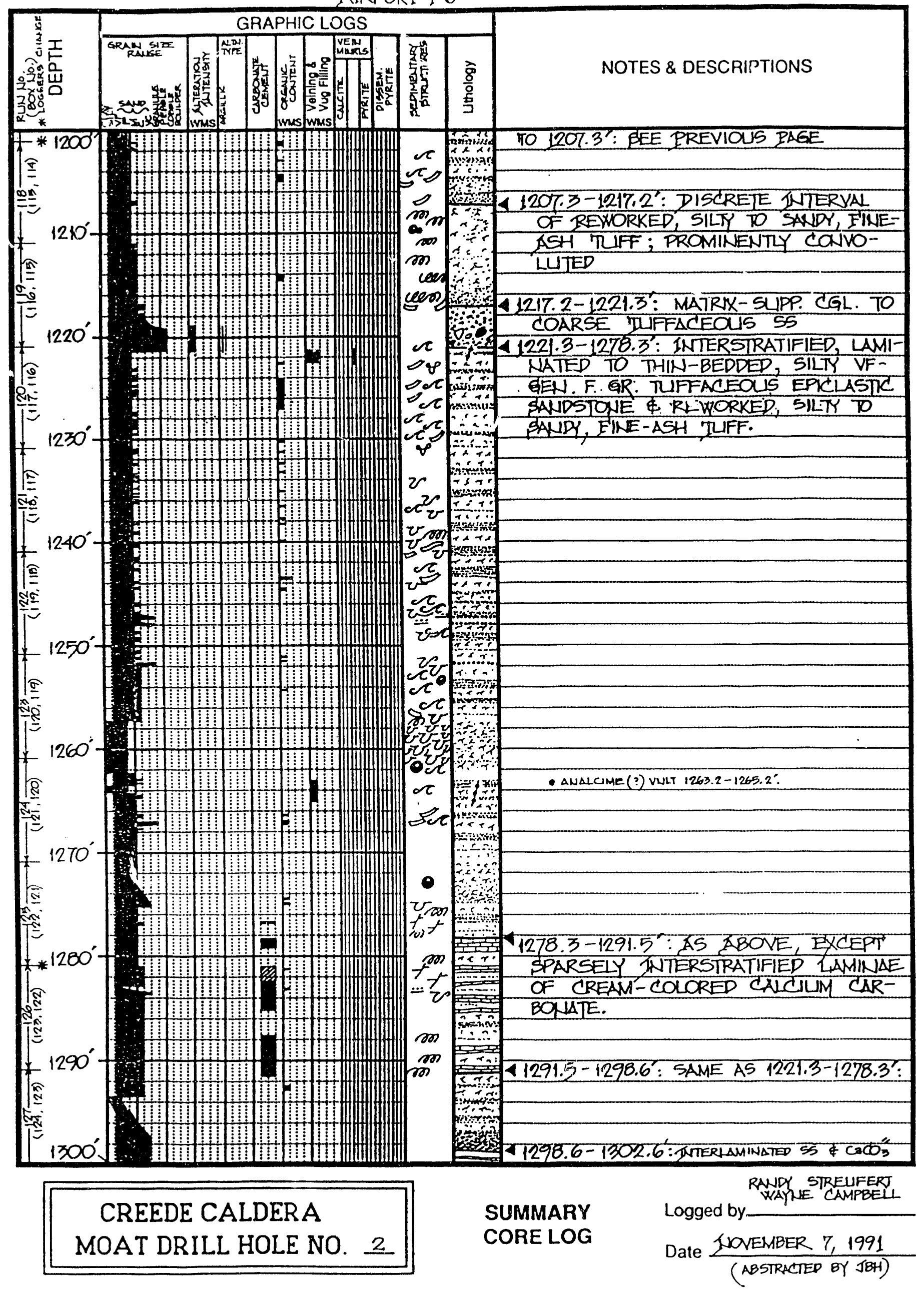




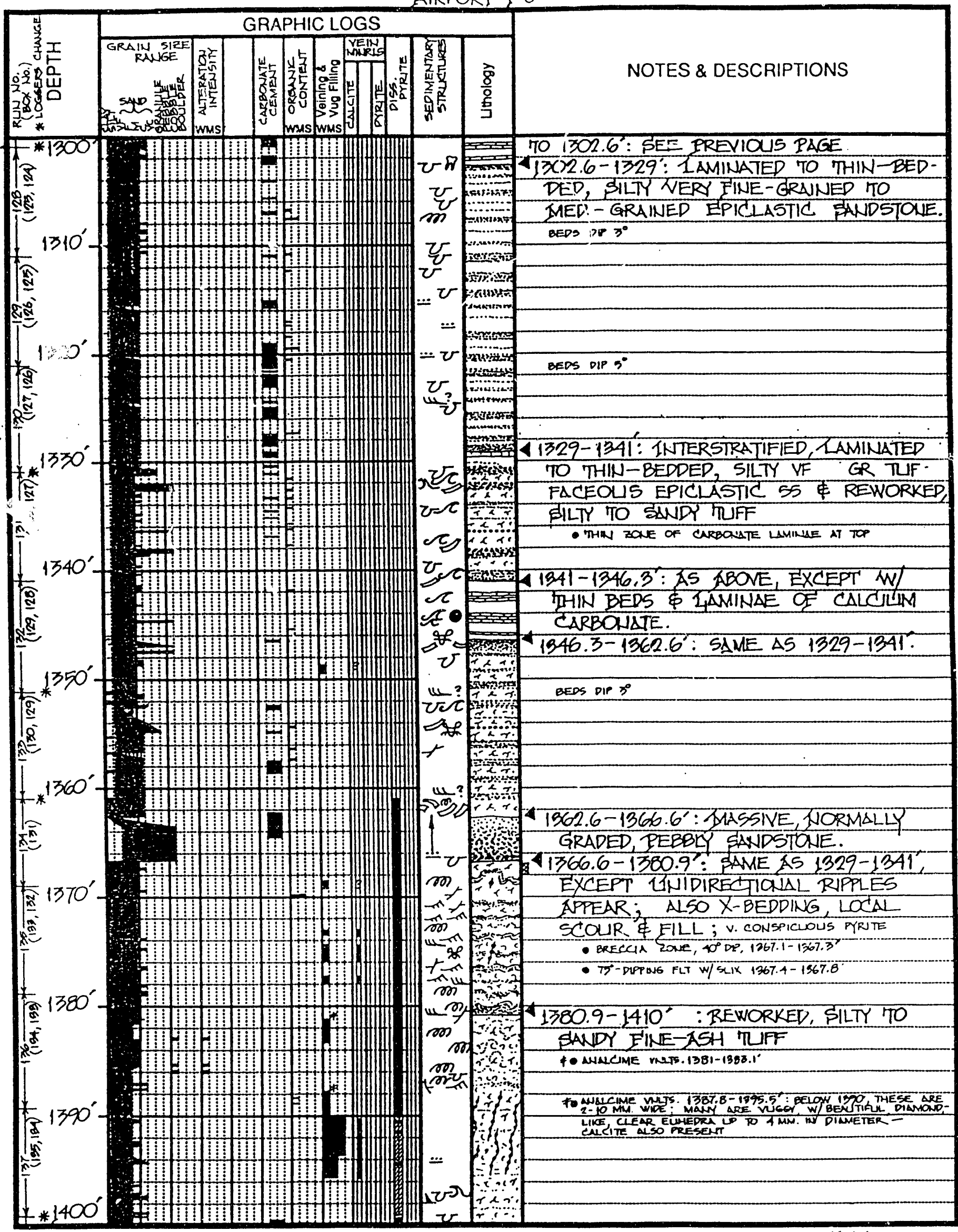

CREEDE CALDERA MOAT DRILL HOLE NO. $\underline{2}$
DAN OHAYCS

Logged by $\frac{\text { WAYME CAMPBELL }}{\text { RAIDY STRELIFEET' }}$

Date NSOVEMBER $7 \& 8,1991$ 

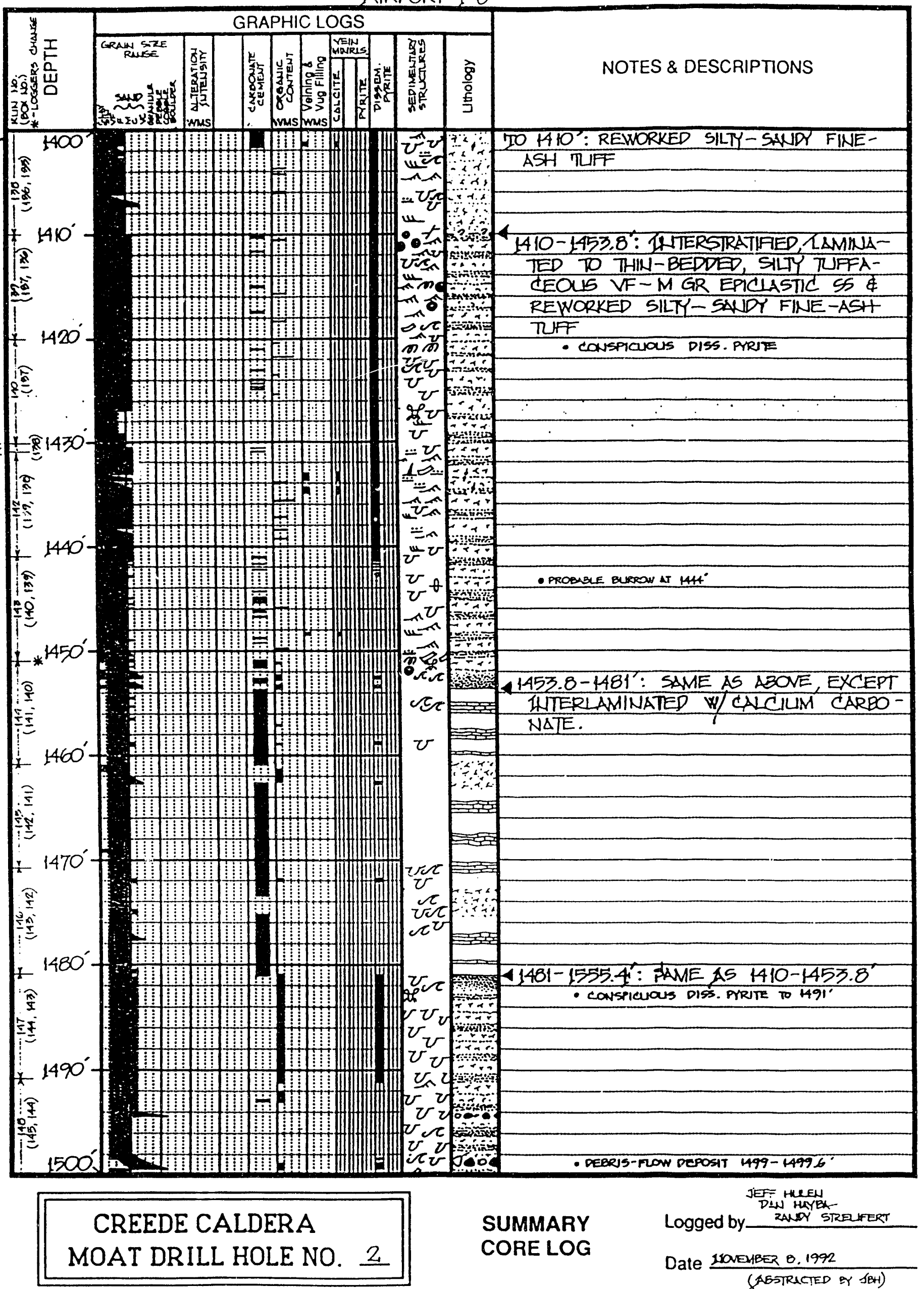

SUMMARY CORE LOG

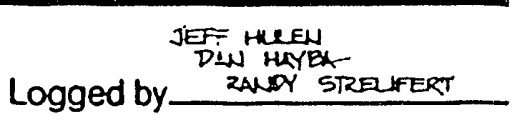

Date INONEABER 0.1992 (AESTRACTED or SBH) 


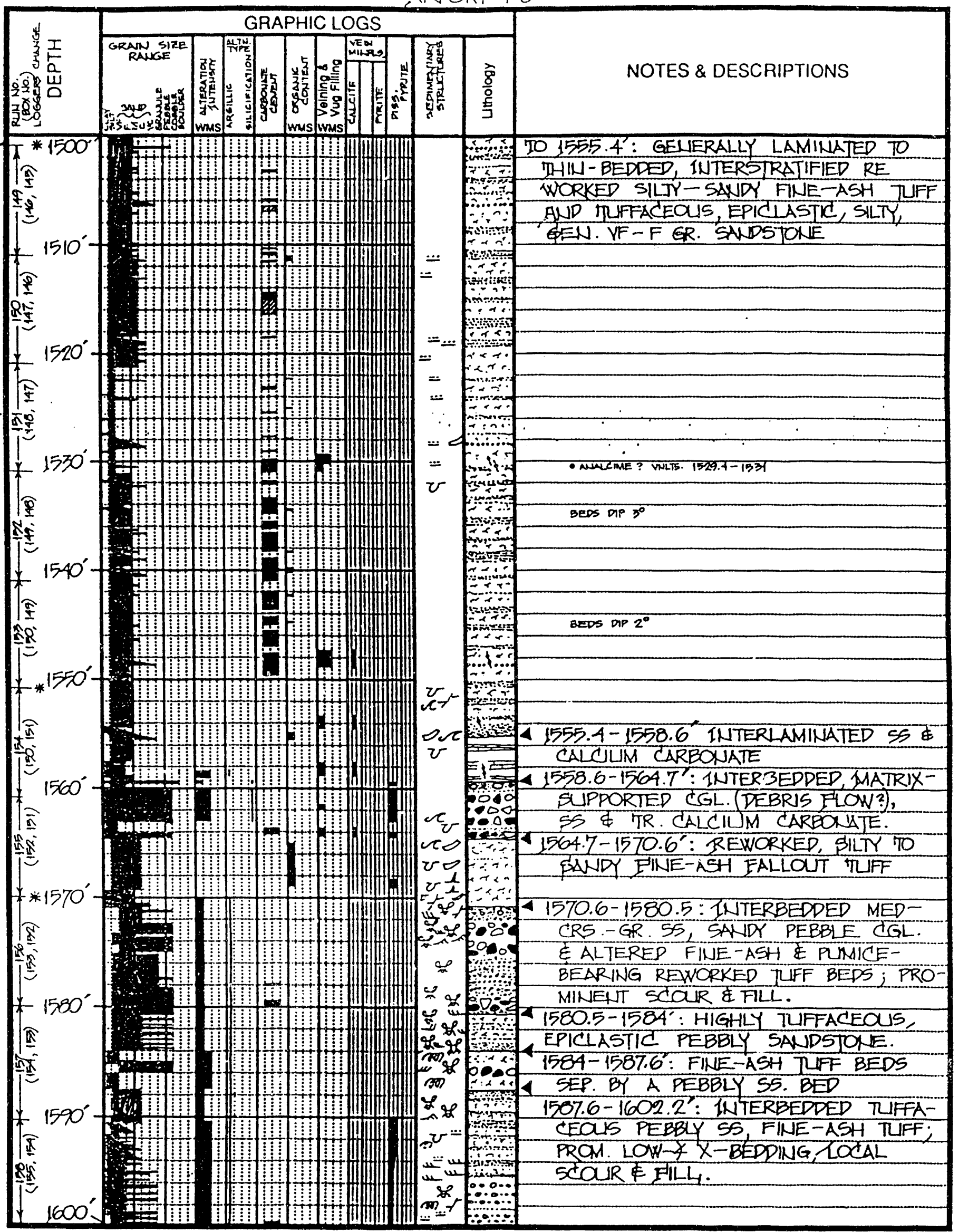

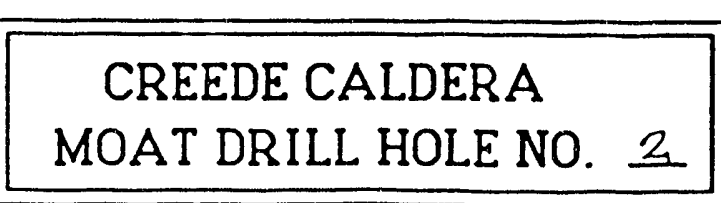

SUMMARY

CORE LOG
WAYNE CNFBELL

PANY STRELRERT

Logged by PANEAYYGA

Date LOVEMBER O-9 1591 


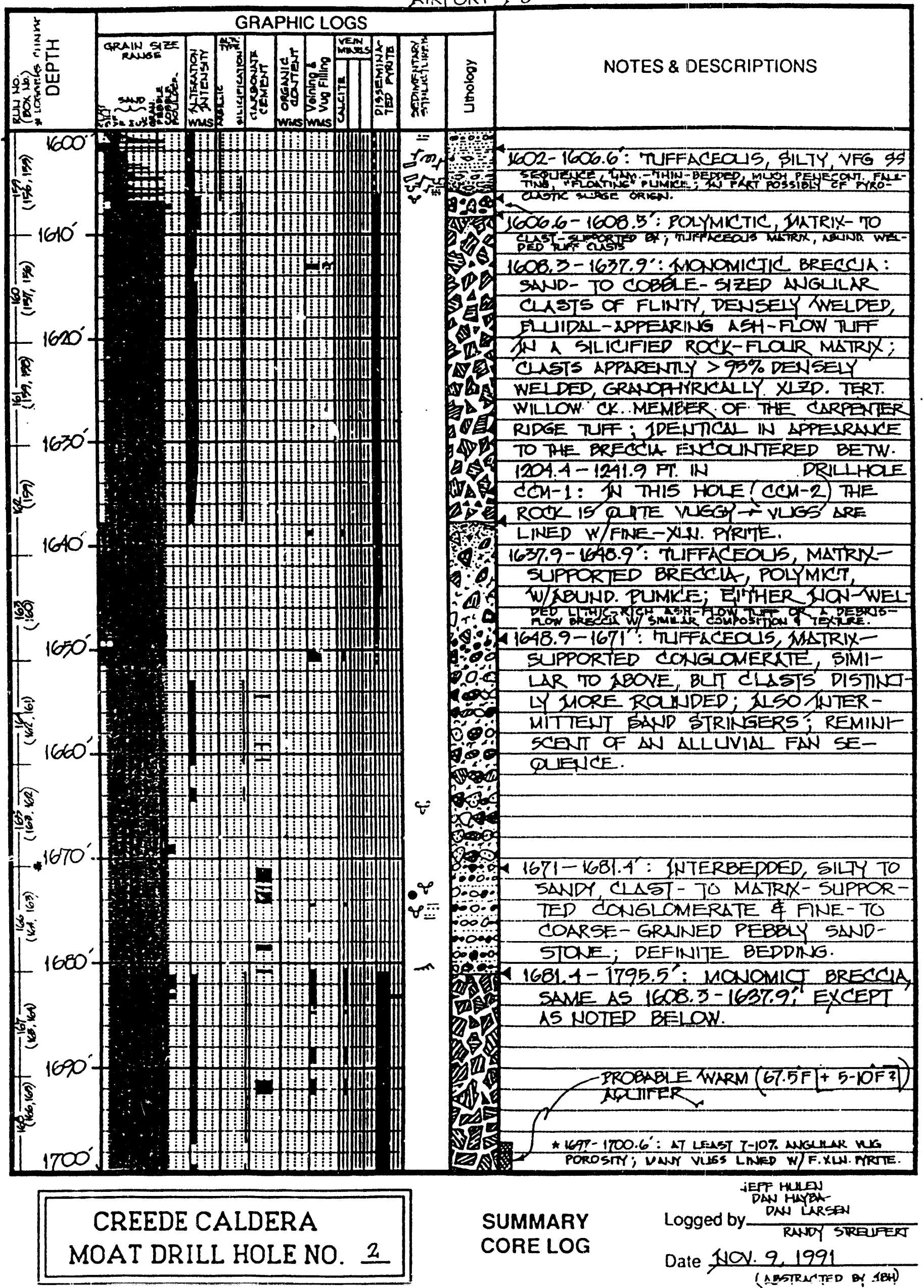




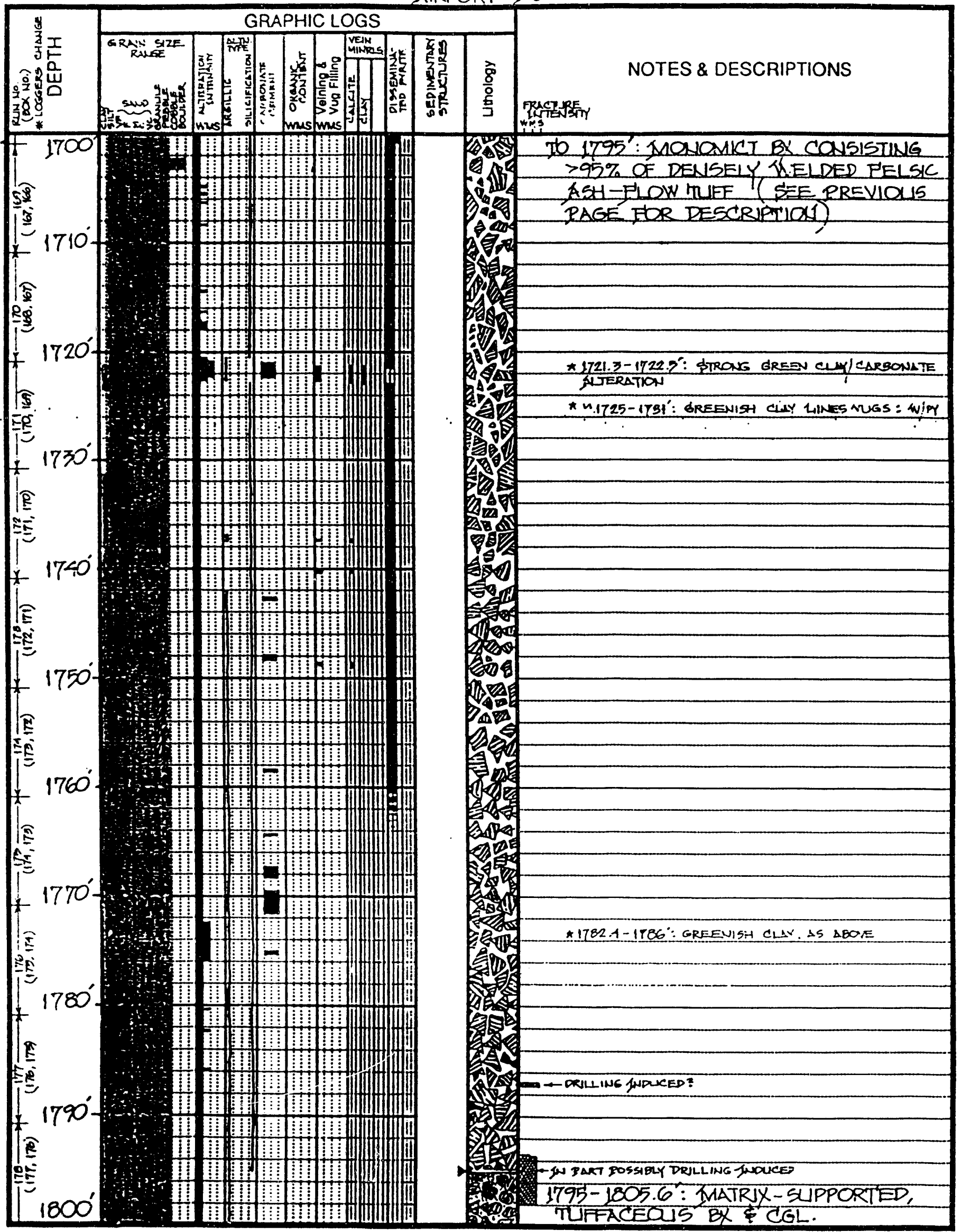

CREEDE CALDERA MOAT DRILL HOLE NO. $\underline{2}$
SUMMARY

CORE LOG
Logged by RUNR RSEN STREUFERT

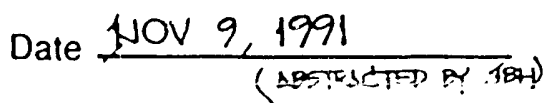




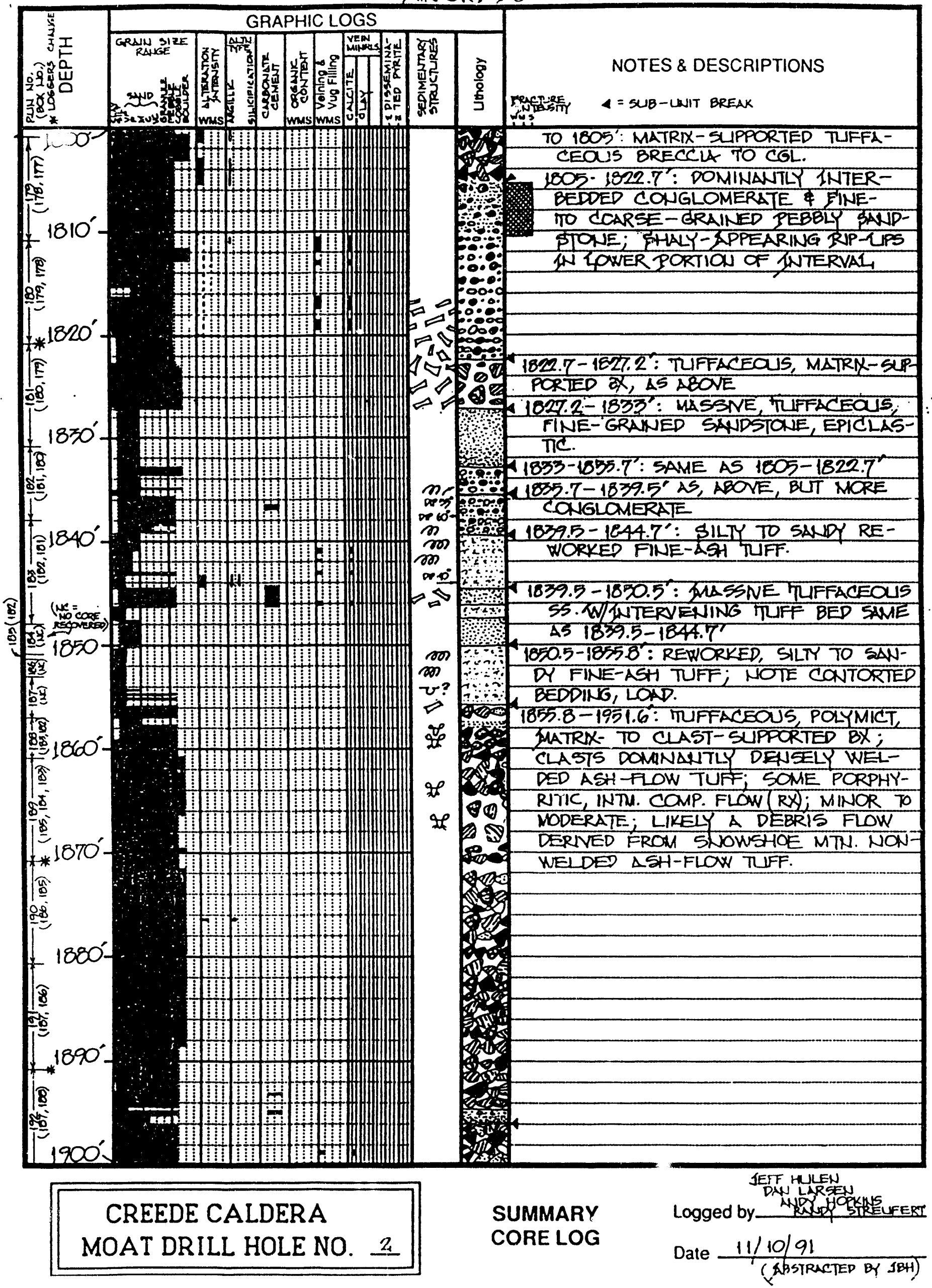




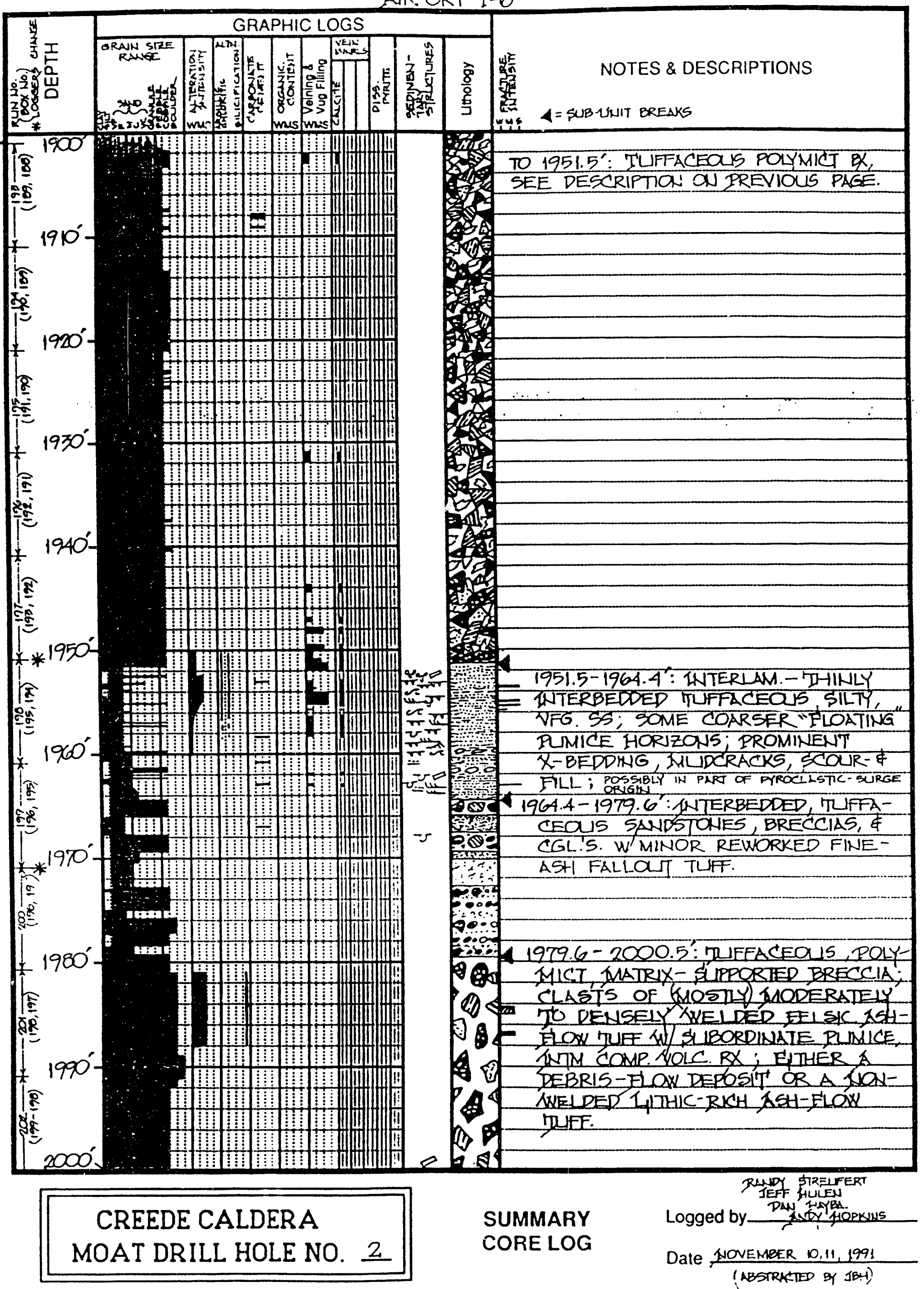




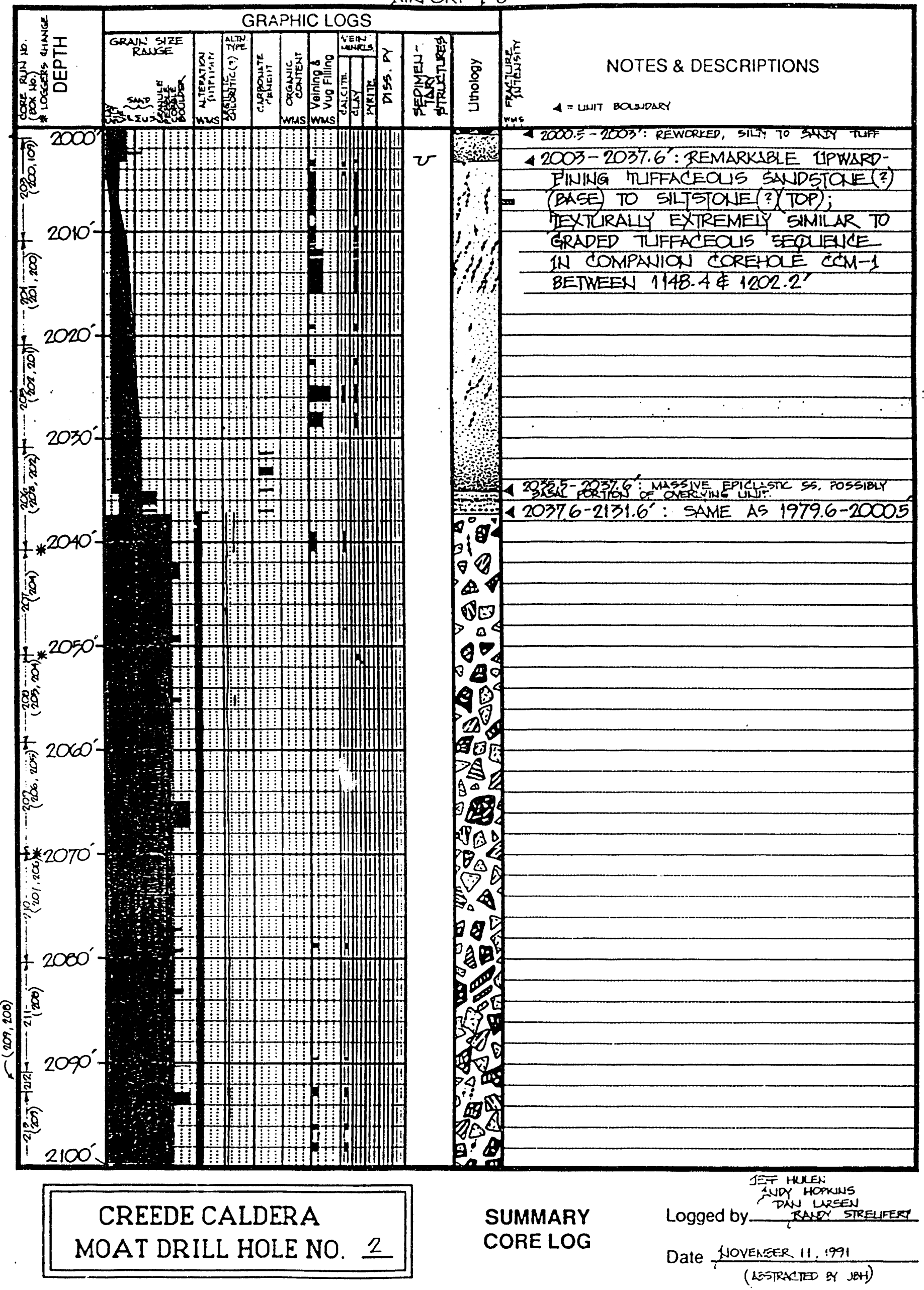




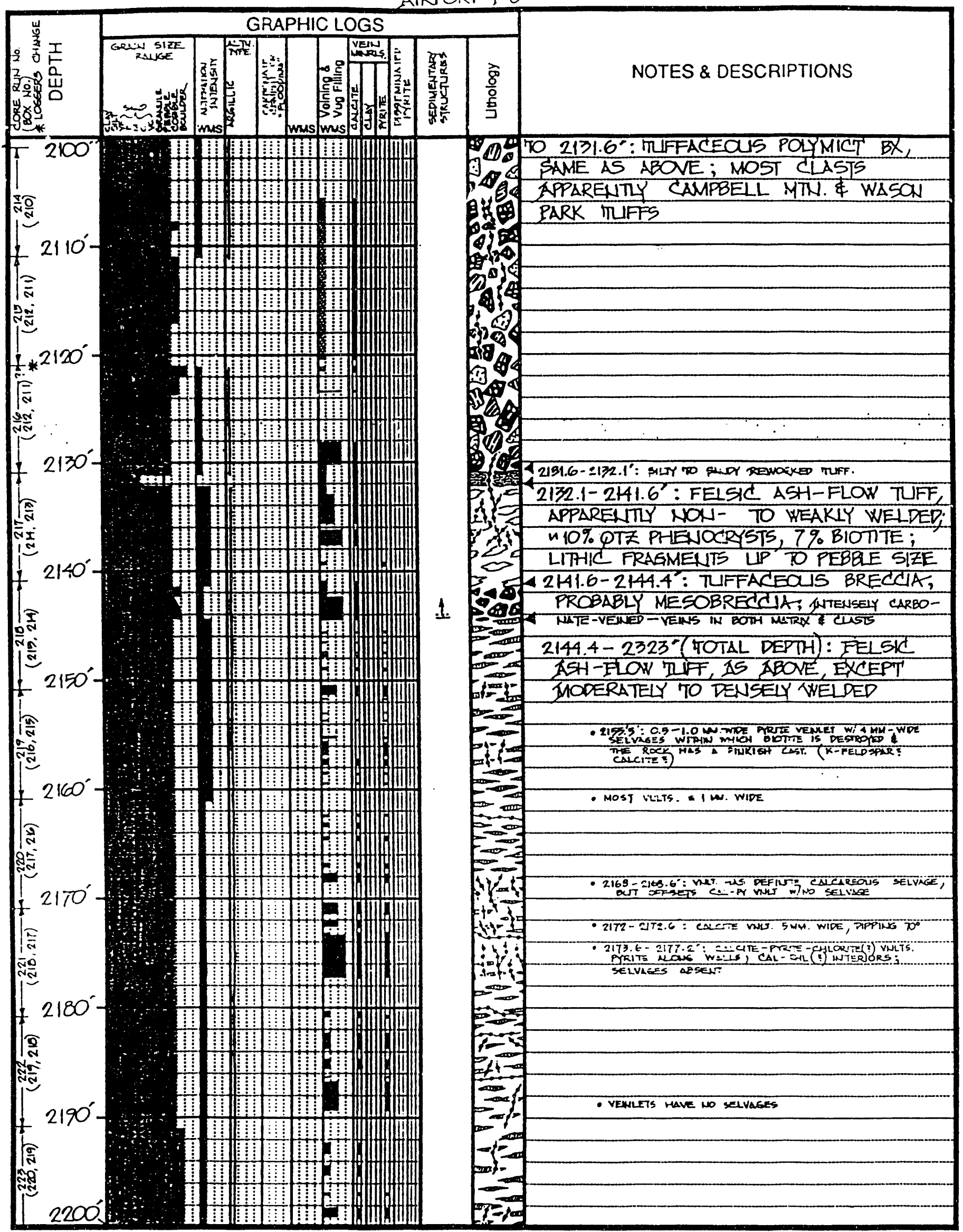

CREEDE CALDERA MOAT DRILL HOLE NO. $\underline{2}$ CORE LOG
Logged by JAN LARSEN \& CANDEREN

Date LOVEABER 12, 1991 


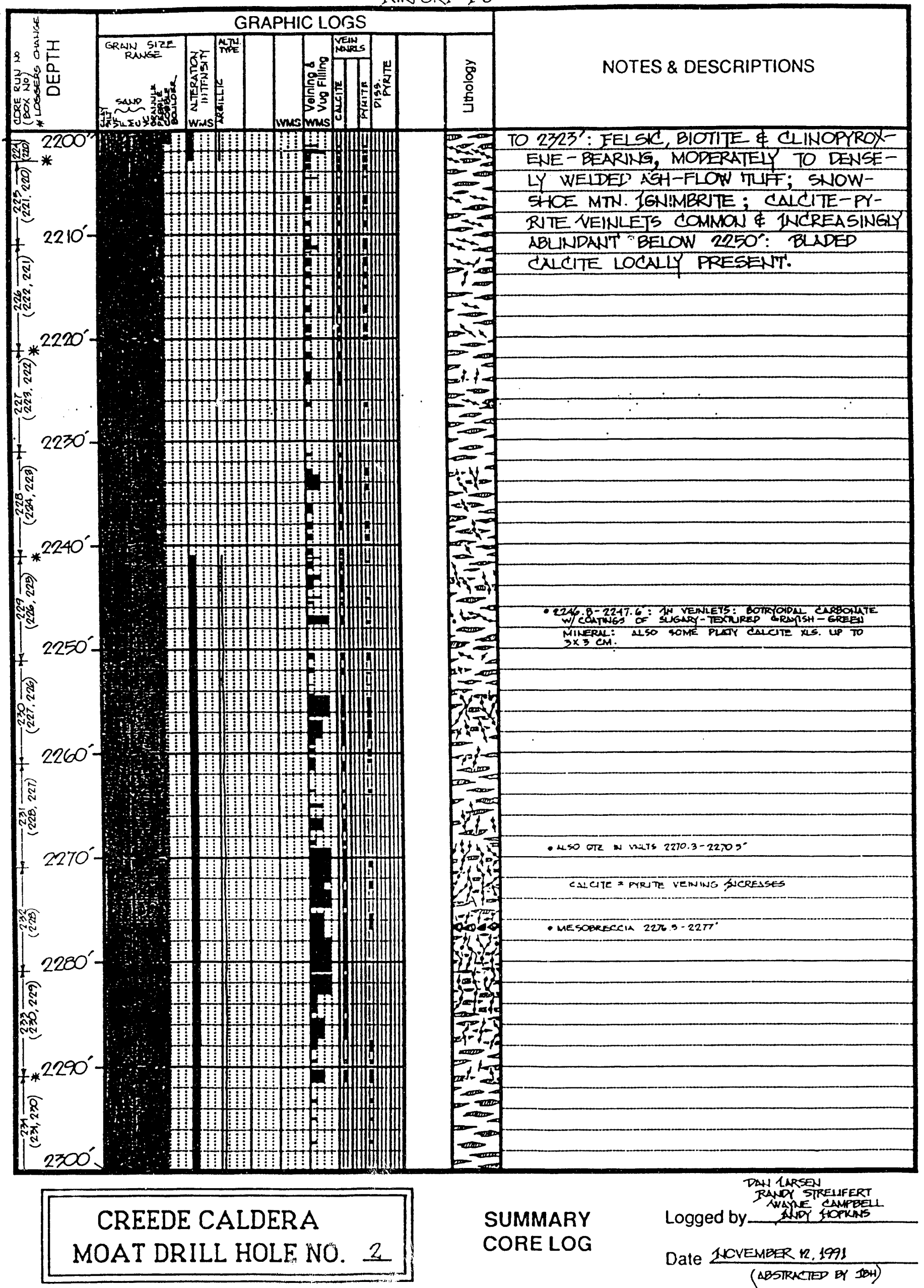




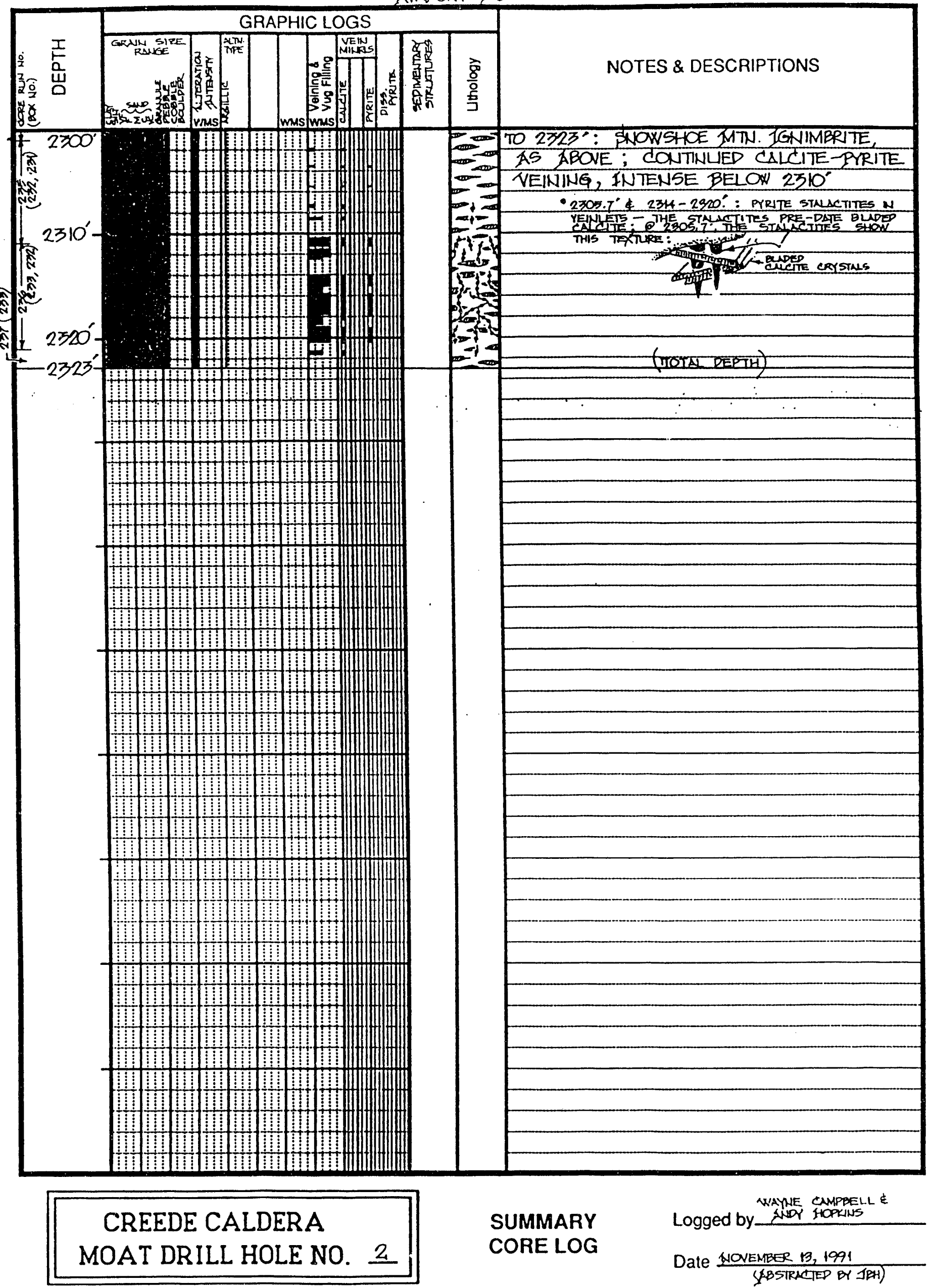




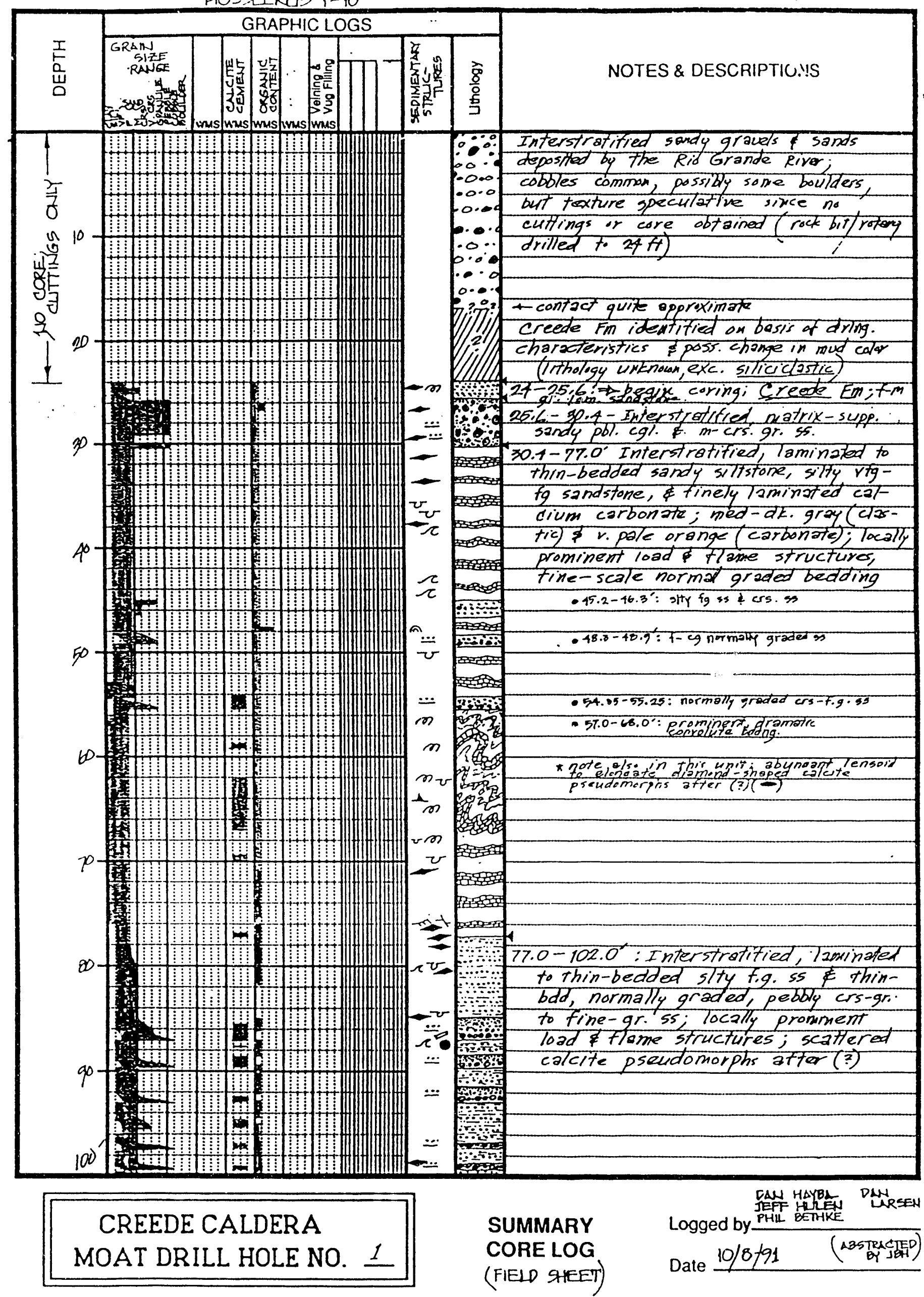



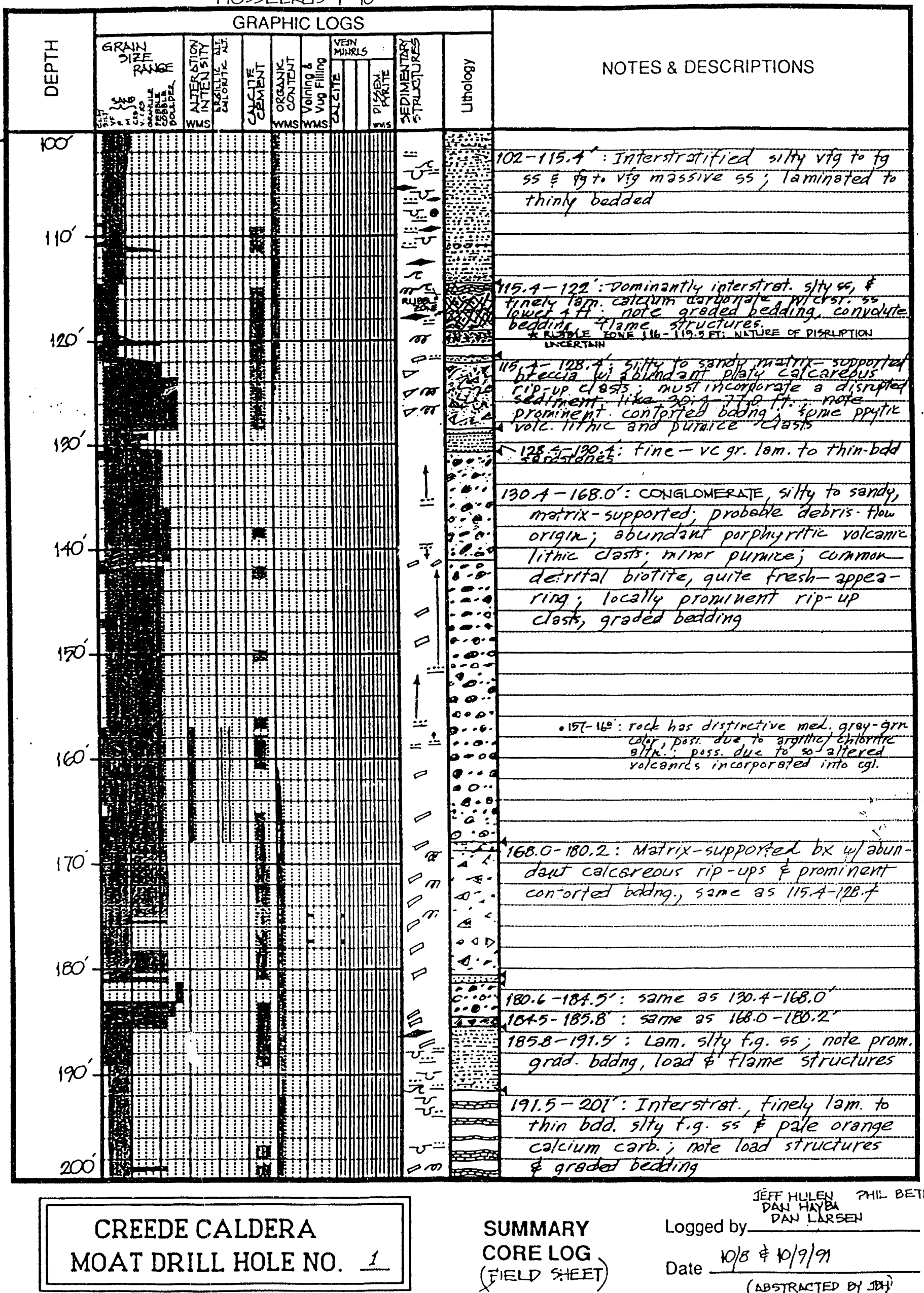

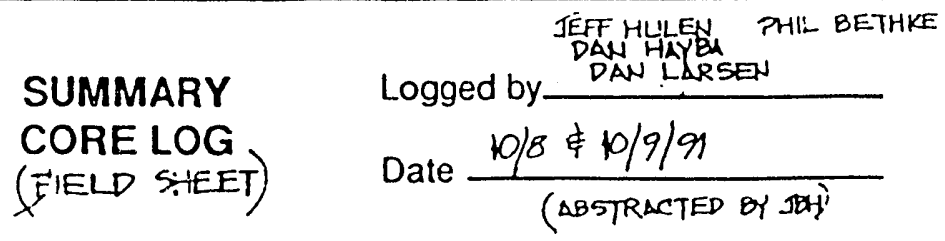




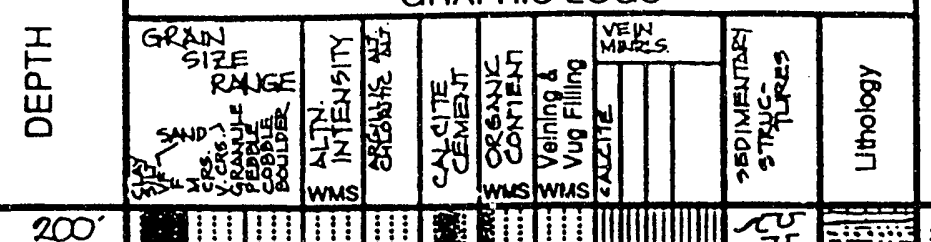

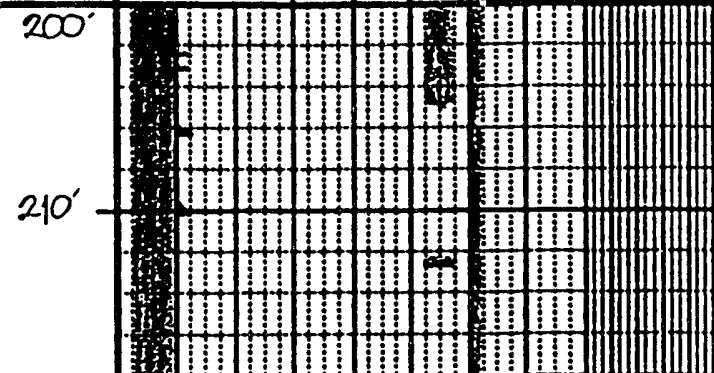

$220^{\prime}$

$1+111$

च्च

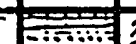

v.

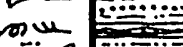

$=0$

$u v$.

然

isition

NOTES \& DESCRIPTIONS

$201-213.8$ ': Alternating intervals of

lam to thin-bdd, slly $15-i g$ SS of

same except w) up to $15 \%$ (vol)

interstratified fincly moingted

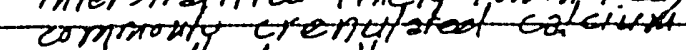

carborato lamellie

च

픈

m

兄它

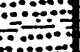

213.8-237.4: Interstrafified, Taminated to thin-bedded silty ta 70 vig 55; 55 is immature, wlabund. detrital bfe, probable plimice, volc. lithic sand grains; orominemt

$\infty$

vo load casts, flame structures, normatt graded bedding of convolute bedding (origin as furblidites probable)

-235.35': probable I crat thick fallowit tent

$230^{\prime}$

$\cos c$

vo

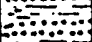

$11+1+1$

- possple burrow $7 t^{7} 225.5^{\prime}$ 管

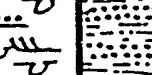

$\checkmark=$

$1+1$

$240^{\circ}+1$

$\dddot{-}$

252

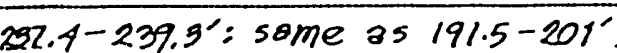

$239.3-241.2^{\prime}$ : same as 213.8-237, w/o

tew Thin calcium carb. Intabeds

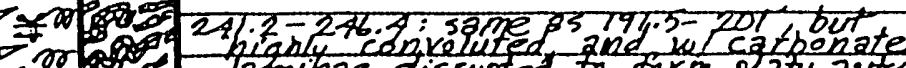

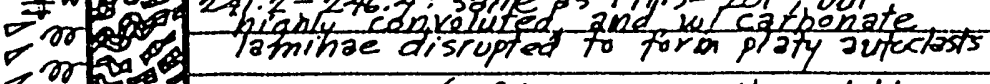

11111010

$246.1-248.5^{\prime}$; fine-gr 55 , thin-bdd

of 248.5-252.7': same 85 191.5-201!

$250^{\prime}+1: 13$

$\frac{\pi}{c}$

$\varnothing$

車

252.7-284.8': silty to sandy matrix-

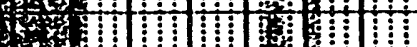

$260^{\prime}$

supported conglomerate to breccia; - probsole debris-flow oriain; anak-

- L lar to subrounded clasts of domi-

- o. nantly porphuritic volcanic rack

$\because$ embedded in 24 immature siltu to

0.0

$0 \ldots$

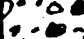

$\because 0 \cdot a$.

$a+\Delta x$

$270^{\prime}$ (10): $:$ :

$\rightarrow \infty$

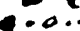

$-a \cdot c$

ro:

0.0

0.0

660.

z

100

284.8-287.3: interstrat. /asn.-Thrn-bdt. (1)

290 '

2... 287.3-291.3: 53me 85 191.5-201

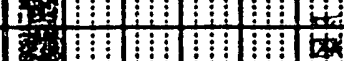

$\therefore=1$ 291.3-296.45:5ame 25 213.8-237.4

$+11$

(1)

$\frac{296.45-299^{\prime} ; \text { massive, normally graded }}{\text { petbly crs-gr to fogr. s5. (immolure) }}$

300

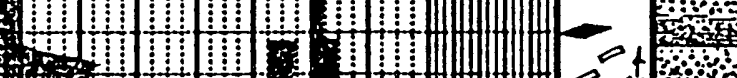

DAN -ARSEN, RANOT

STRE UFERT, NORA FOLEY,

CREEDE CALDERA

MOAT DRILL HOLE NO. 1
SUMMARY

CORE LOG

(FIELD SHEET)
Logged by 资EF HUULW

Date $\frac{10 / 9-10 / 19 / 91}{\text { (ABSTRAC }}$ 

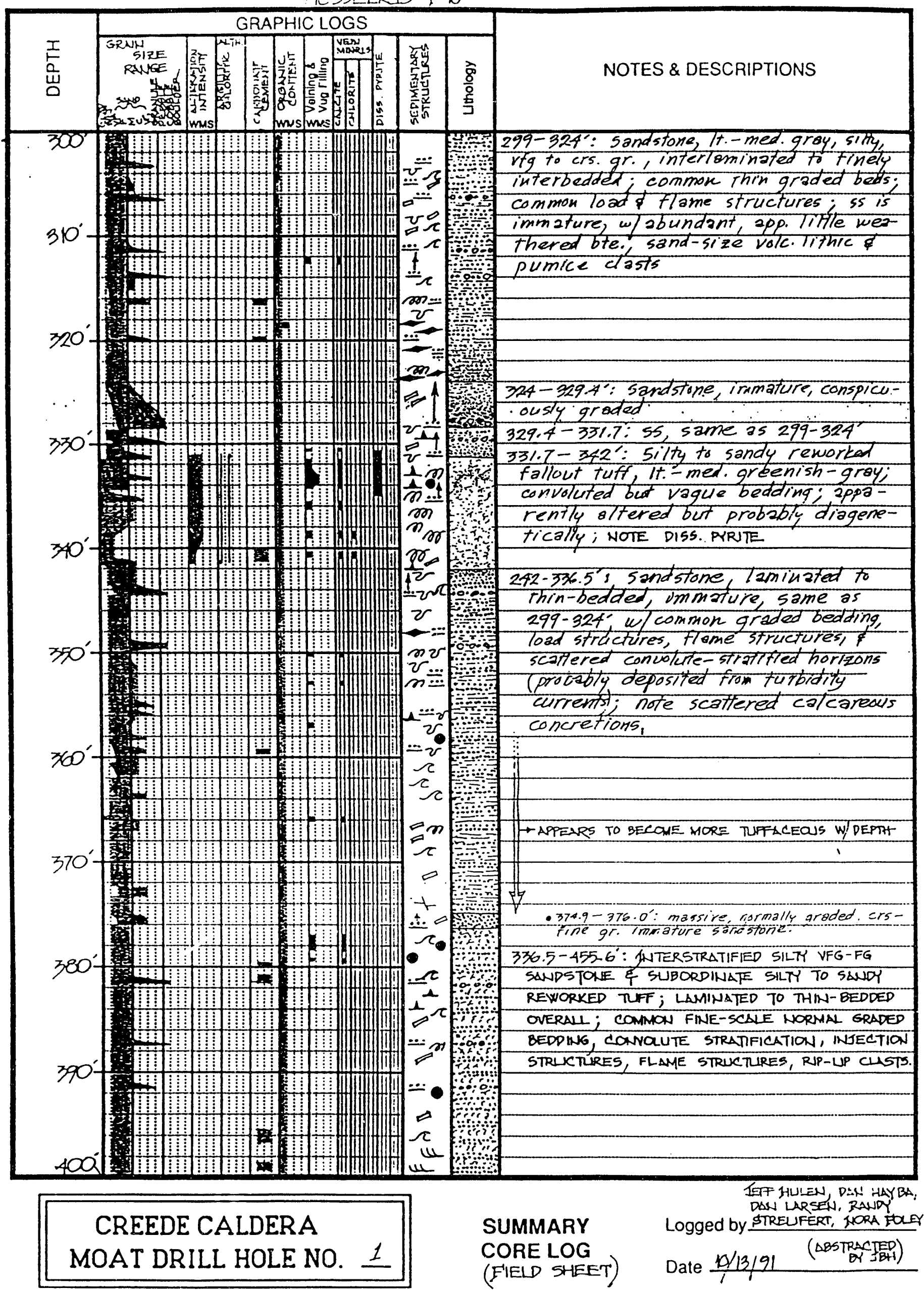

IEF HULEN DEN HAYBA. DAN LARSEN, RANDY

SUMMARY

CORE LOG

(FIELD SHEET)

Logged by STREUFERT, KORA FOLF

Date $10 / 3 / 91$ or $13+1)$ 
GRAPHIC LOGS

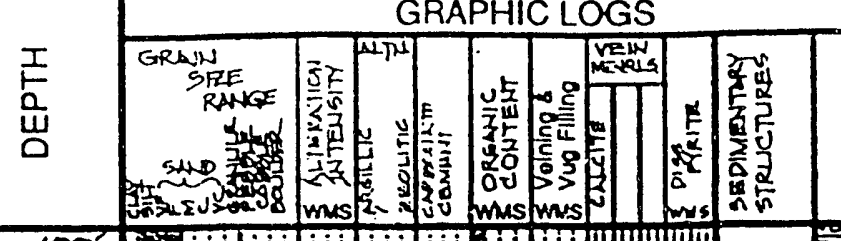
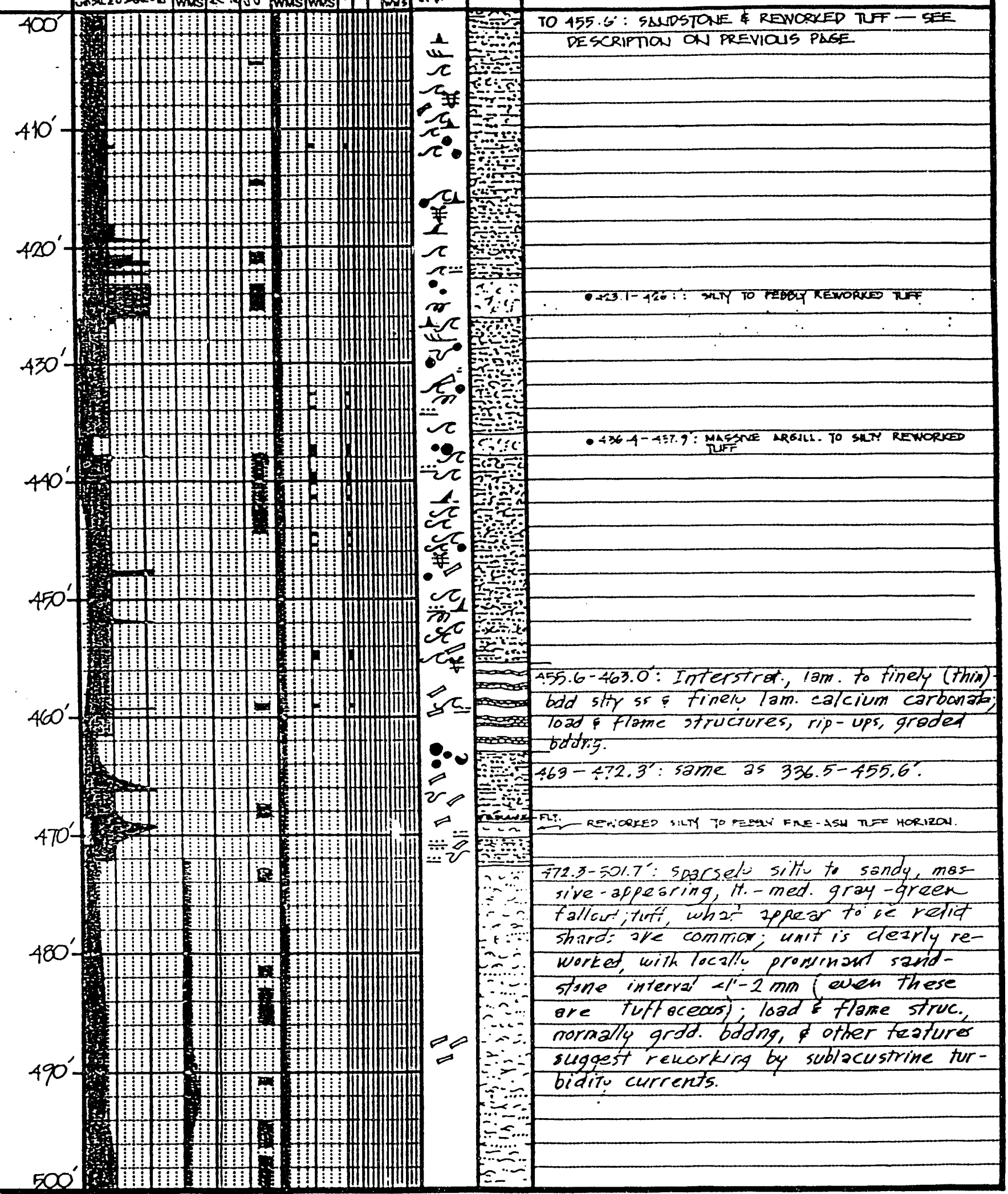

500 '

总

NOTES \& DESCRIPTIONS

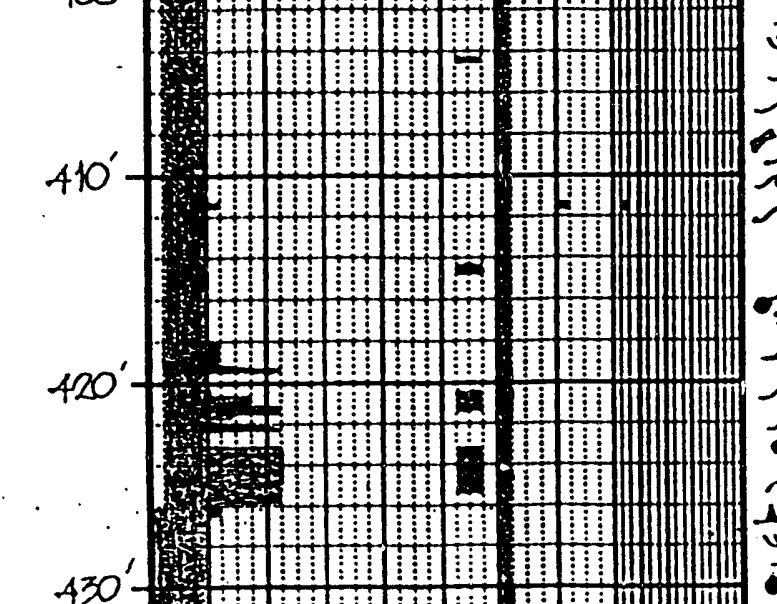

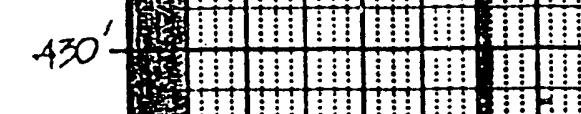

$490^{\prime}$

$1: 1: 1$

1

10

:

(n)

1: 1

(1)

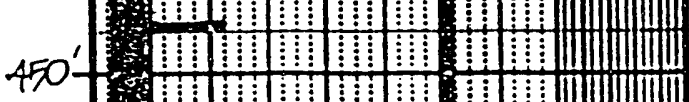

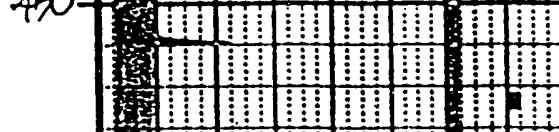

$460^{\prime}$

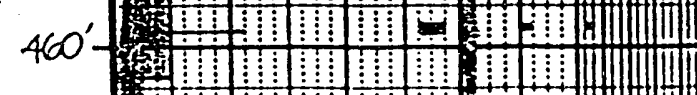

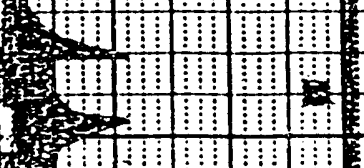

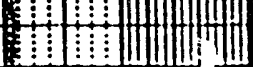

in

$480^{\prime}$

$1: 1: 1$

:

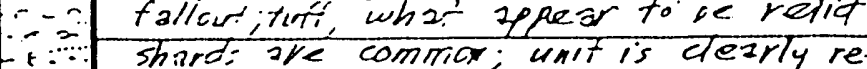

:-:- shard: ale commin; unit is clearly re-

:-. stone interra' $41^{\prime}-2 \mathrm{~mm}$ (even these

$\therefore$ :.: are fuffoceast; load f flame struc.

$\therefore=$ rormally grdd. bddng, other features

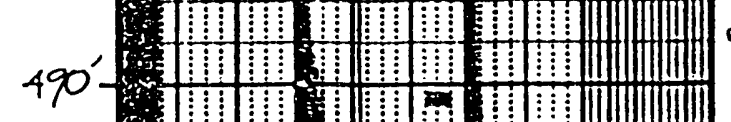

$\therefore$ sugdest reworkirg by sublacustrine fur-

$\therefore$ biditu currents.

$=-:$

$=-1$

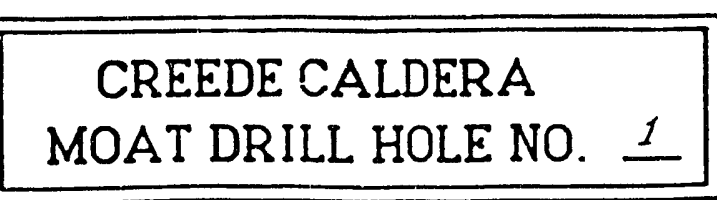

SUMMARY

CORE LOG

(PELD FHEET)
DAU HAYBS JEFF HULEN, Logged by MORA FOLF, RMUDY FERT

Date $\frac{1 q / 15-10 / 4 / 91}{(\text { SBSTRACTED OS SBH) }}$ 
Page 6 or 15

HOSEELKUS $1-10$

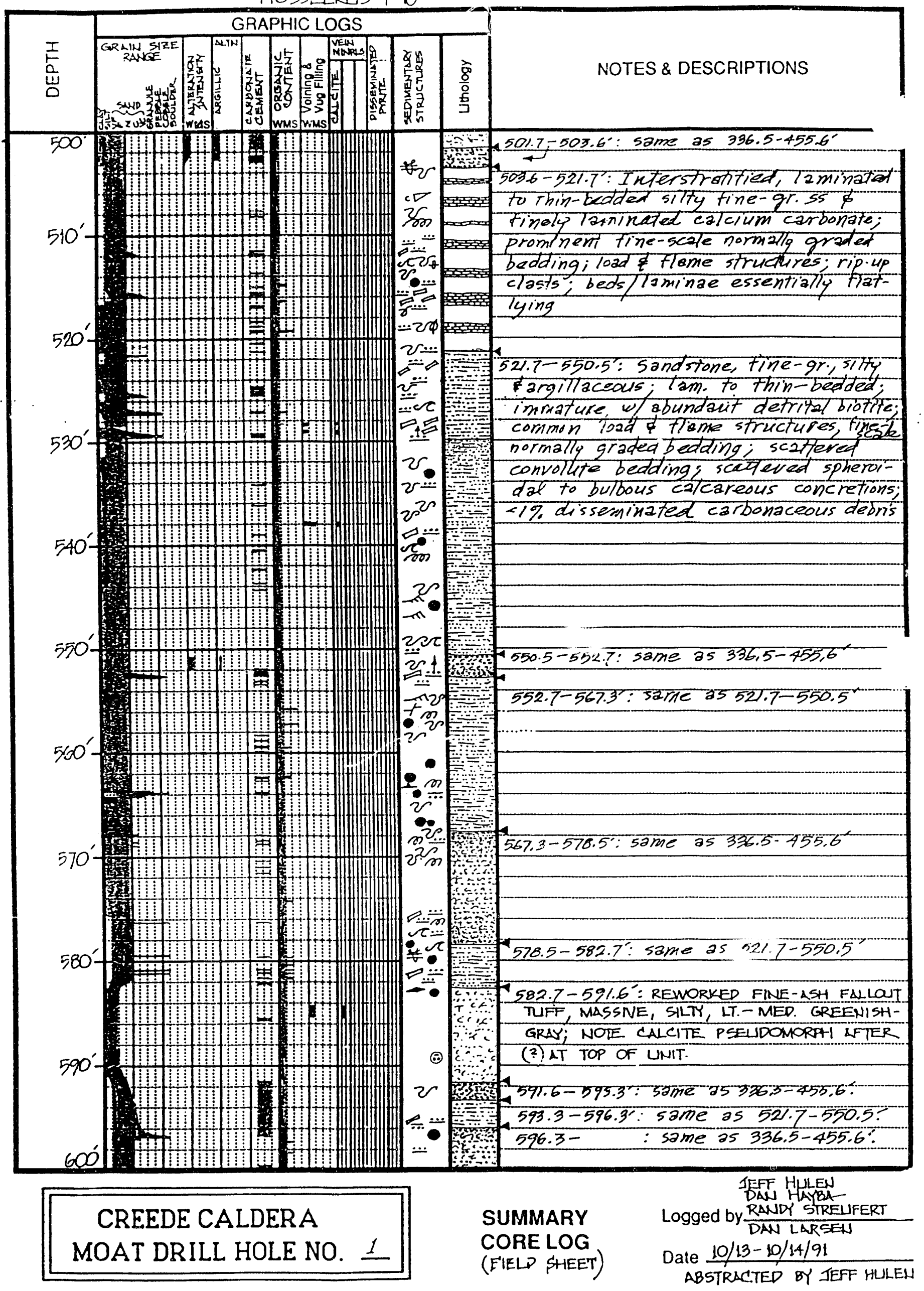


HOSSELKUS $1-10$

Page I of 14

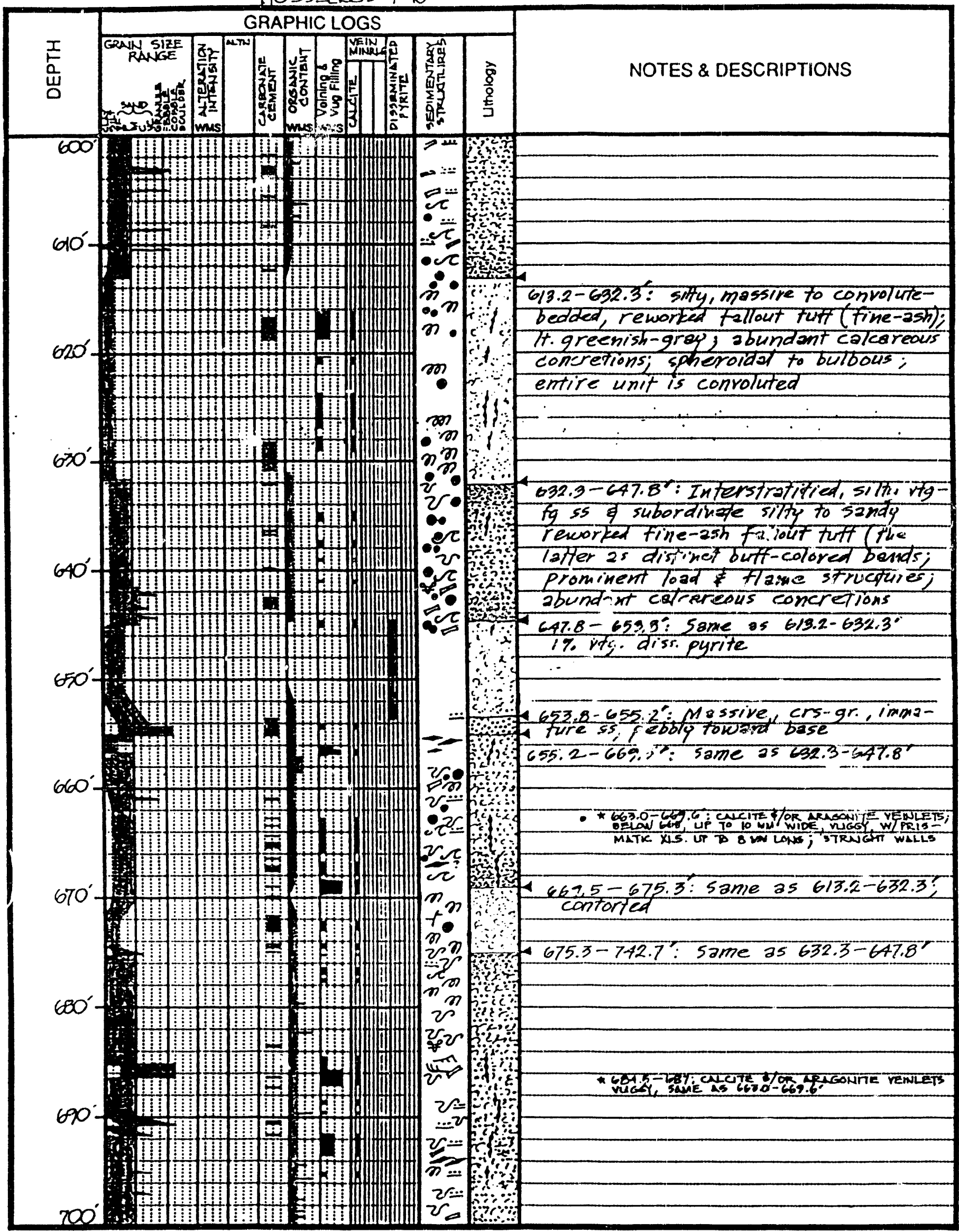

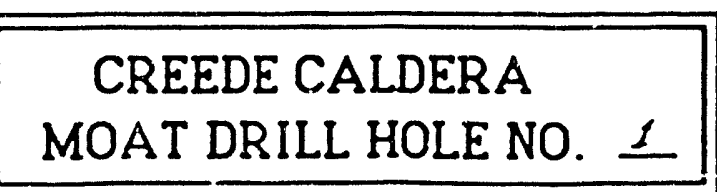

RANDY STREUFERT

DAN LARSEN

SUMMARY

CORE LOG

(FIEID \$HFFT)
Logged by JEFF HULEN

Date $10 / 44-10 / 15 / 91$

(ABSTRACTED OY J.HLLEN) 


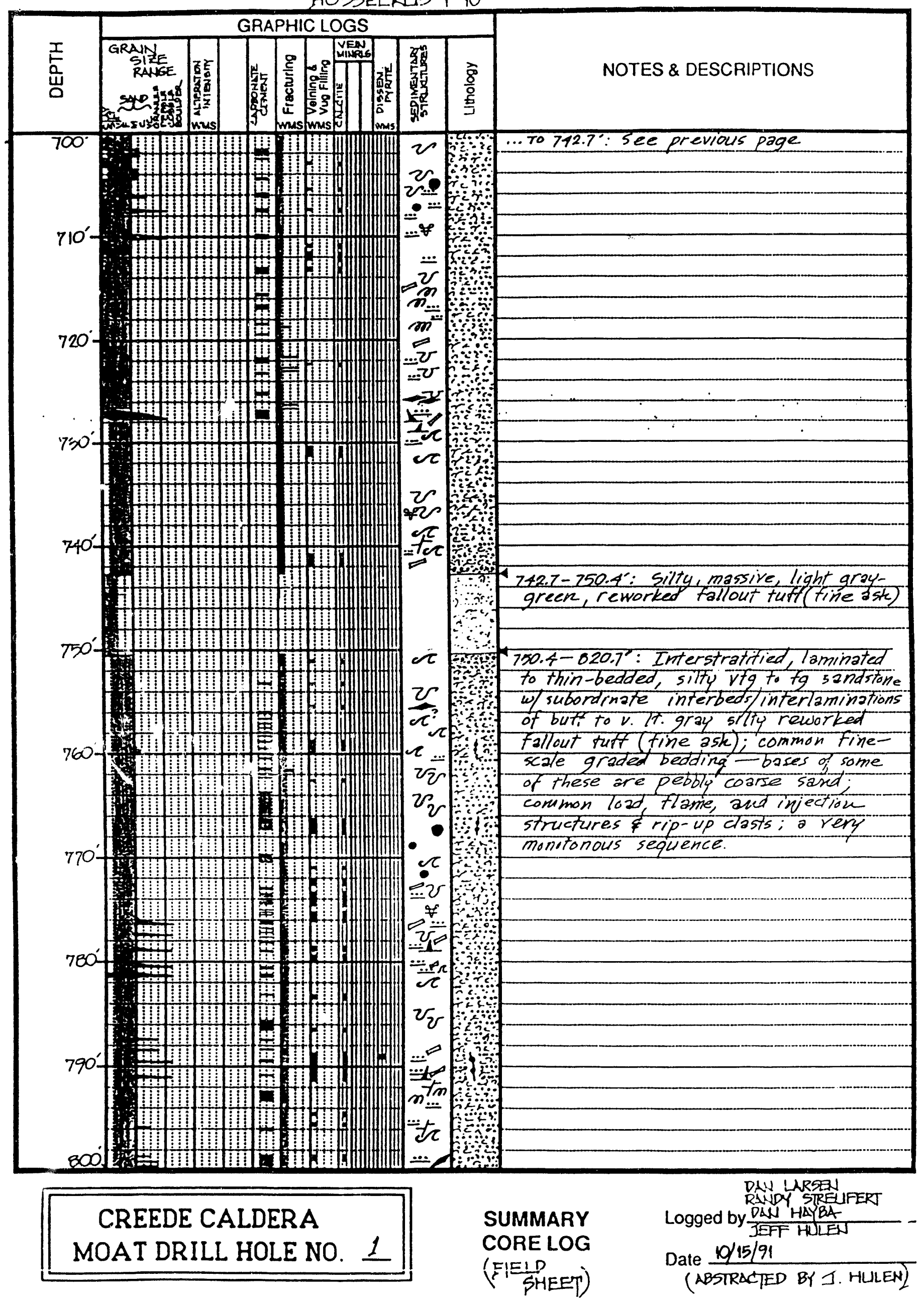




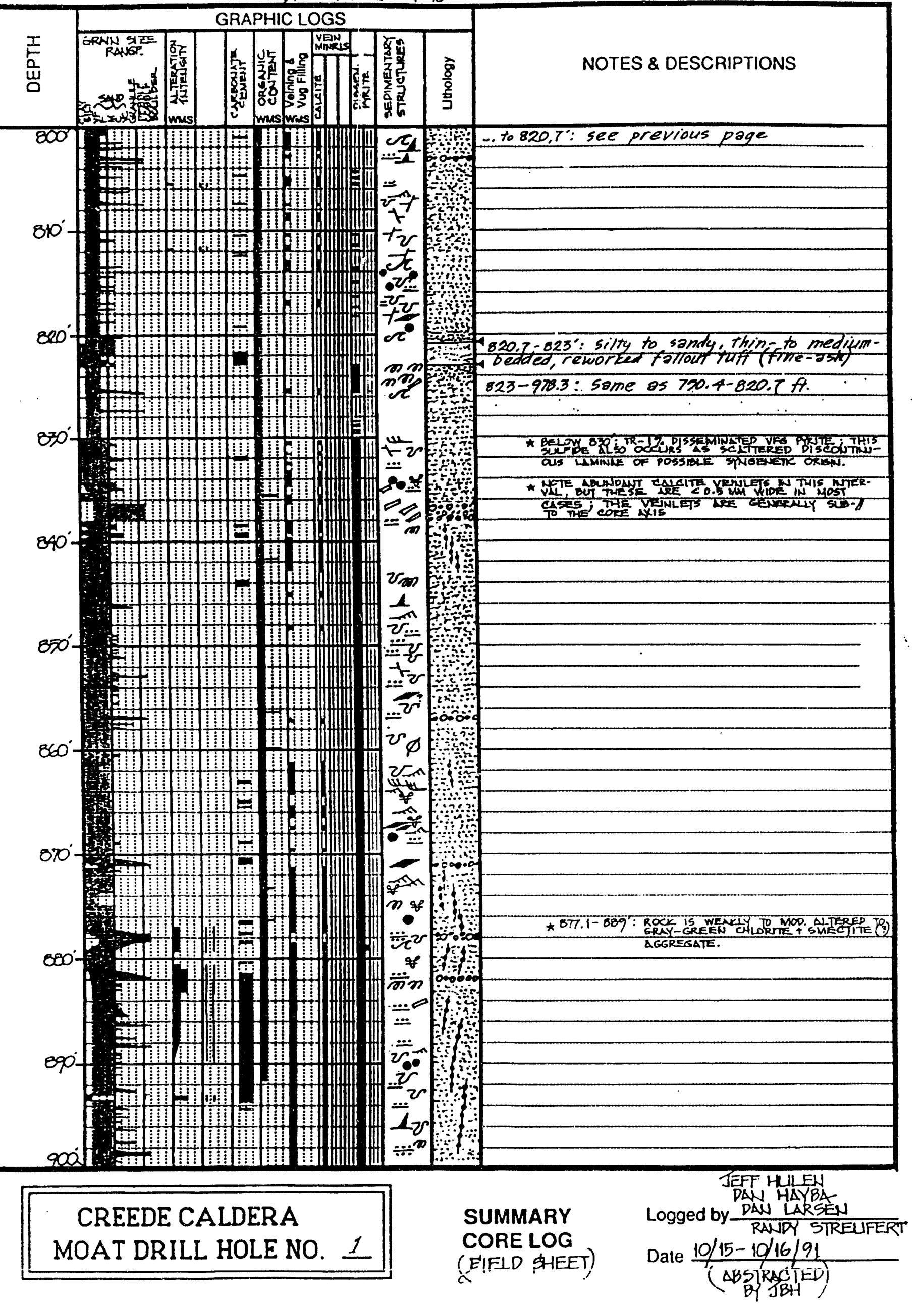




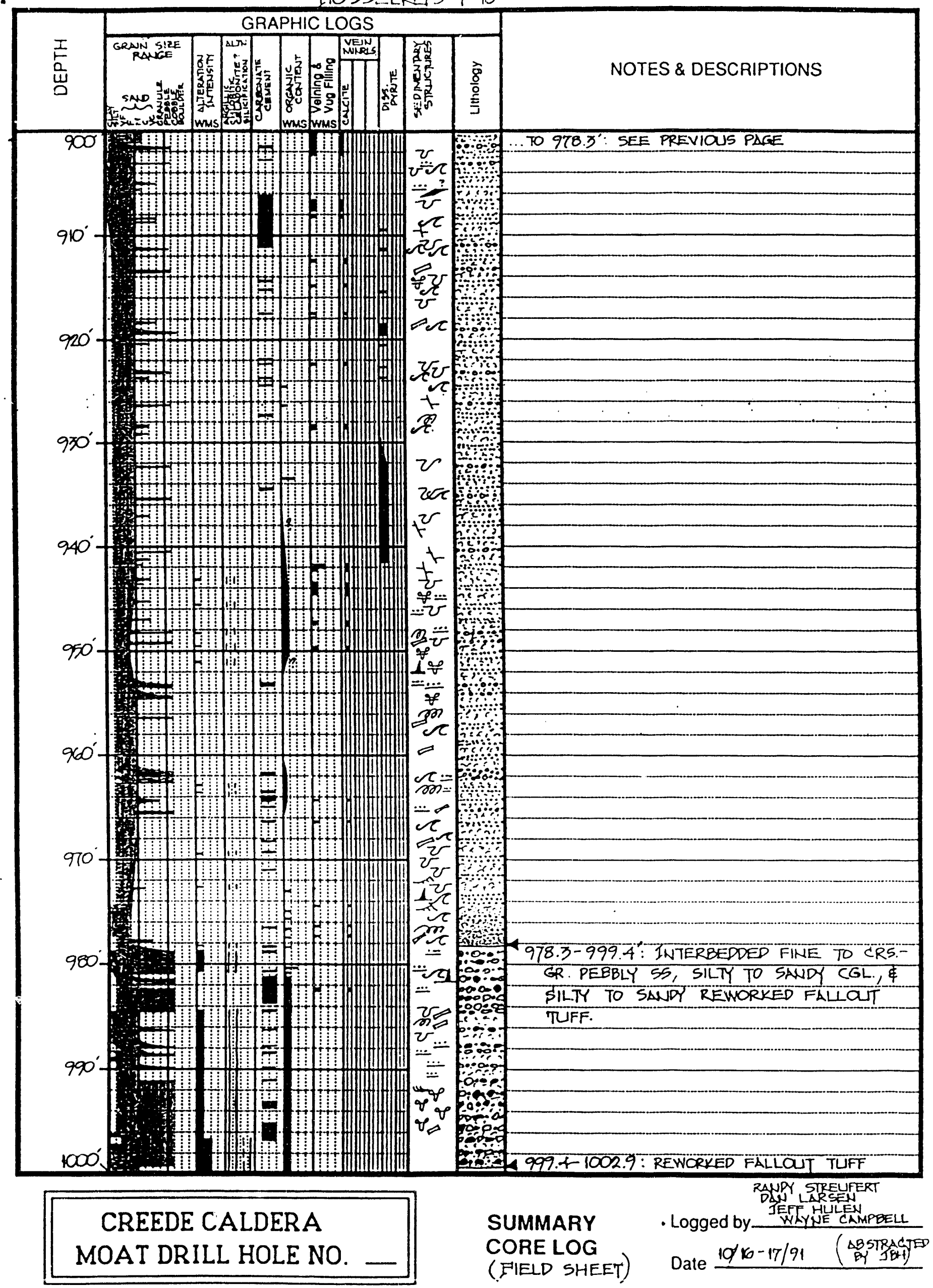




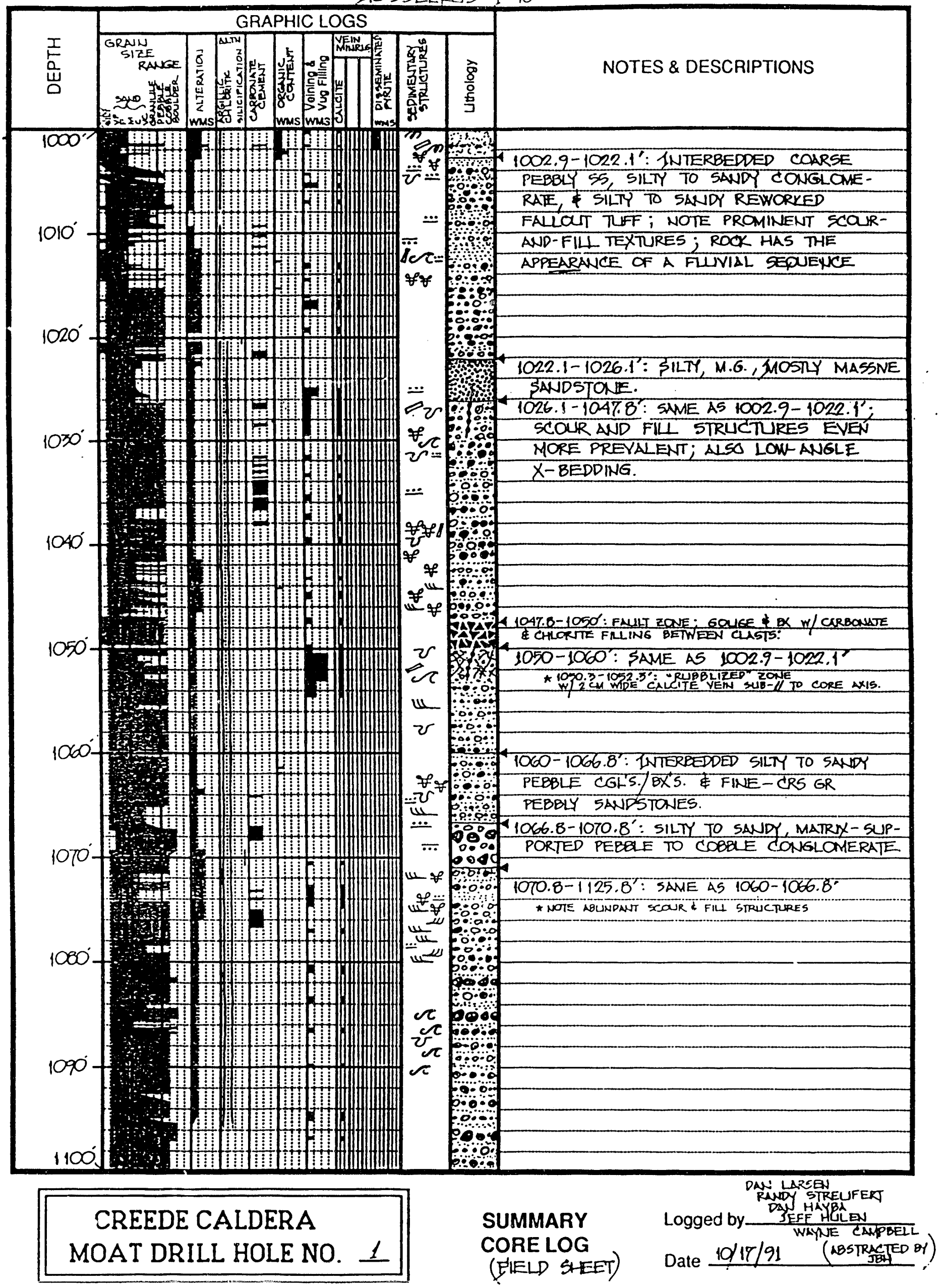


GRAPHIC LOGS

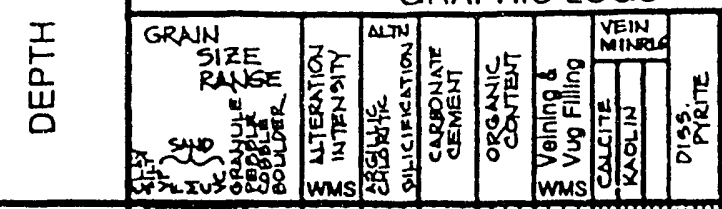

1100 1.

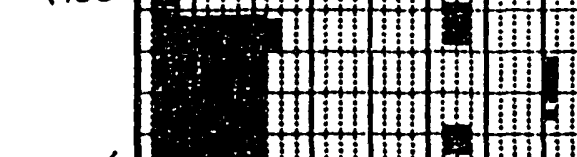

$1110^{\prime}+1110+1$

(1)

1120'

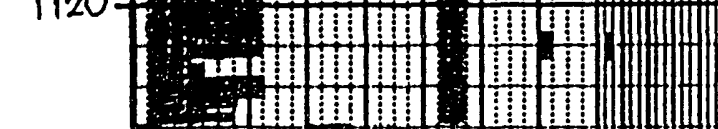

$1130^{\prime}$

$1 !$

II:

1130

1140 f

$1+1+1: 11: 1: 1$

111

$11+1,11$

$1+1$

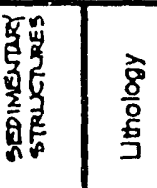

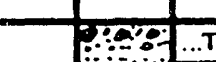

...TO 1125.8': INTERBEDDED SILYY TO SANDY PEBBLE

CONGLOMERATES/BRECCIAS \& FINE-CRS GR

PEBBLY SANDSTONES; LOW-ANGLE X-BEDDING

$\forall$

…

F

ul

$00: 00$

\%5COLR FILL STRLICTURES PRESENT; RX

5.0 .0

- $0 \div$

15 TMMATURE, W/ABLIND. BTE, FSP, SIIT

i.jo

70

...

ibo:

00.

(n)

$\therefore \%$

1125.8-1148.4': REMARKABLE DOWNWARD-

COARSENING TRFFACEOUS CLASTK SE-

QLENCE; ASHT LAMINATED SILTSTONE $\triangle T$ TOP, GRADING DOWNWARD DO BASAL PEBBLE CONGLONERATE; NOTE RIP-LPS.

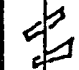

4

1.

$1150^{\circ}$

P

$1: 1: 1: 1: 1: 1$

$11: 1: 111$

$1: 1: 1:$

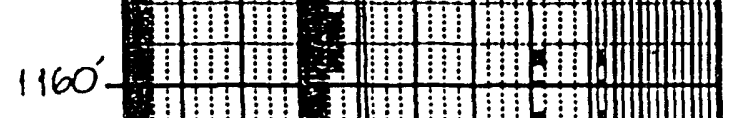

a.

$\therefore$

1148.4-1202.2: REWORKED, SILY TO SAND

$\therefore$ FINE- $\therefore$ SH FNLOUT TUFF; SLIBTLE DOWN-

$\because$ WARD COARSENING OF CLLSTK FRACTION

$\because \because \cdots$

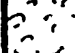

(1)

$$
\text { - }
$$

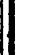

ruxi

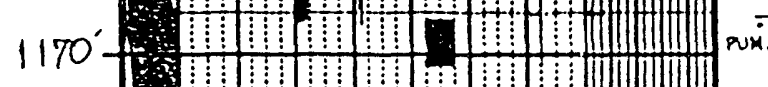

$+7$

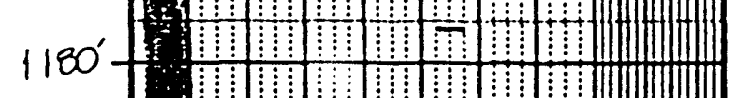

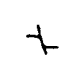

$+$

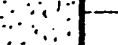

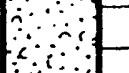
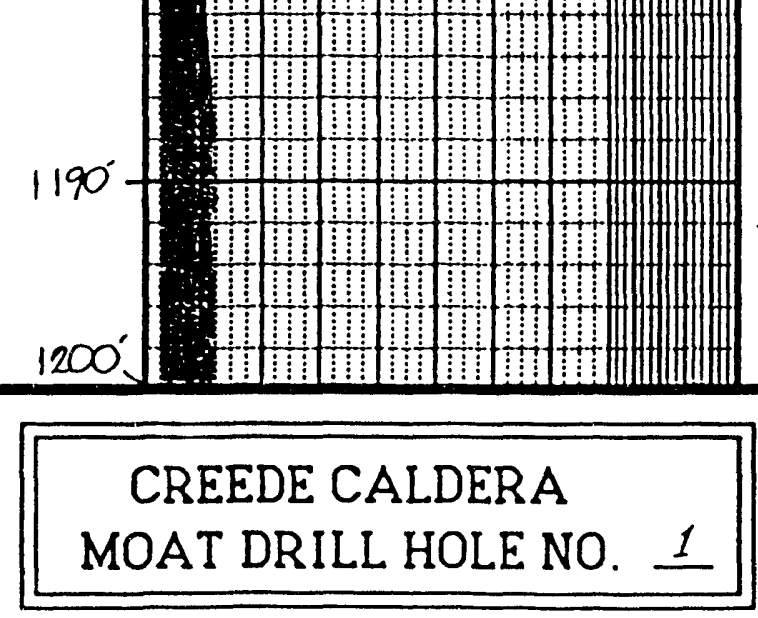


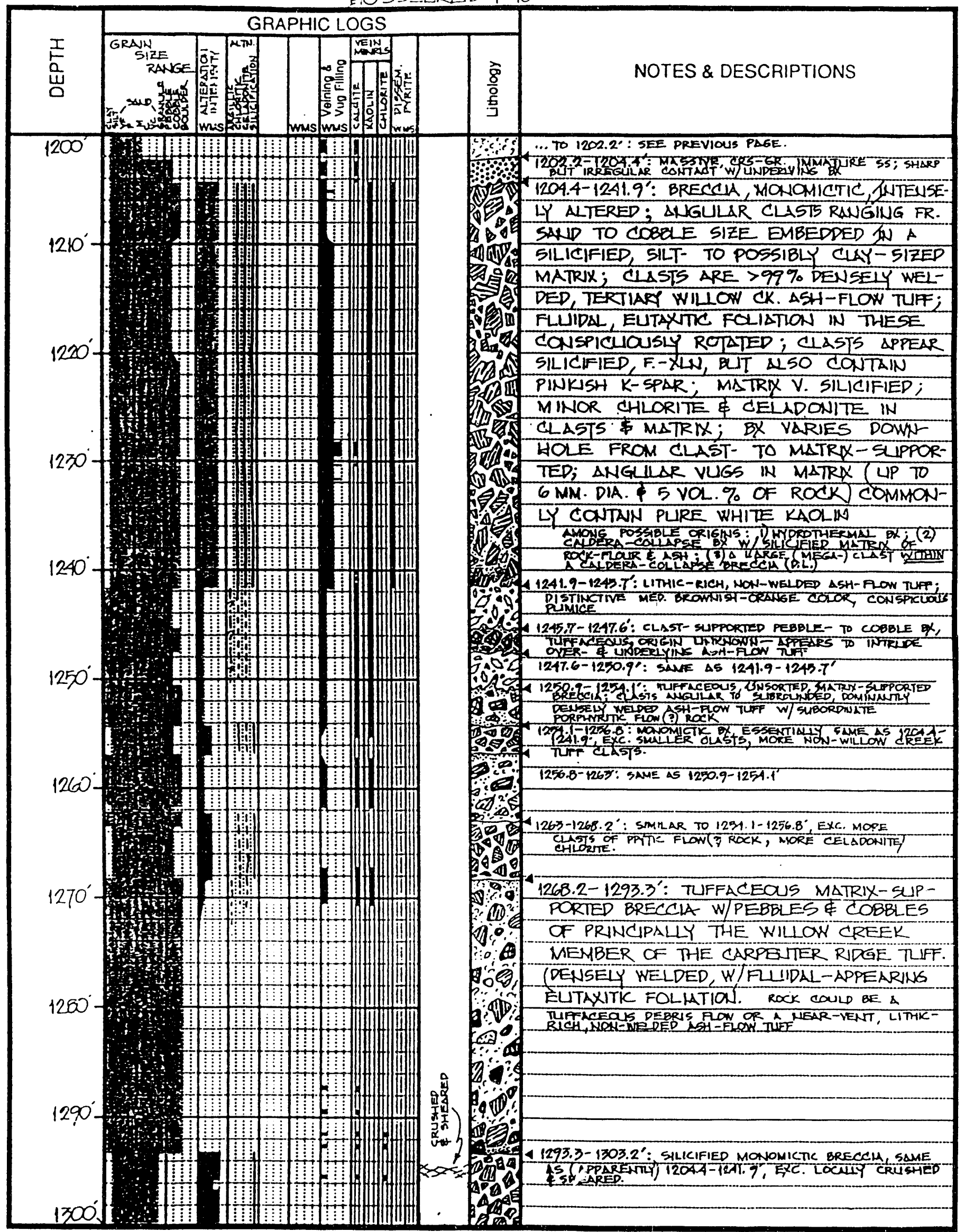

CREEDE CALDERA MOAT DRILI HOLE NO. 1
SUMMARY CORE LOG (E'IELD PHEET) Logged by WEFE HUIIEN Date $\frac{10 / 18-10 / 19 / 91}{(\triangle B S T R A C T E D \text { BY JBH) }}$ 


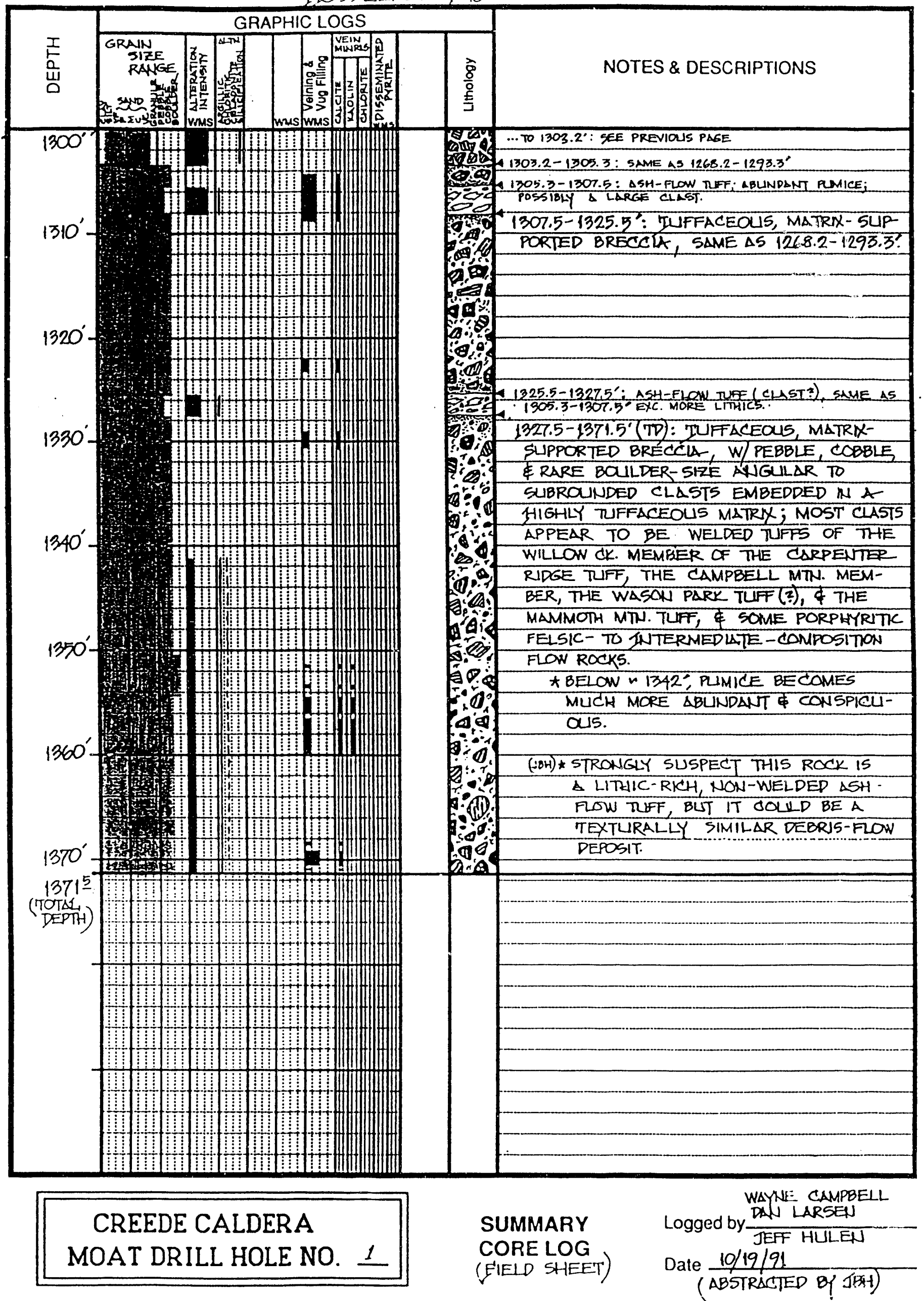



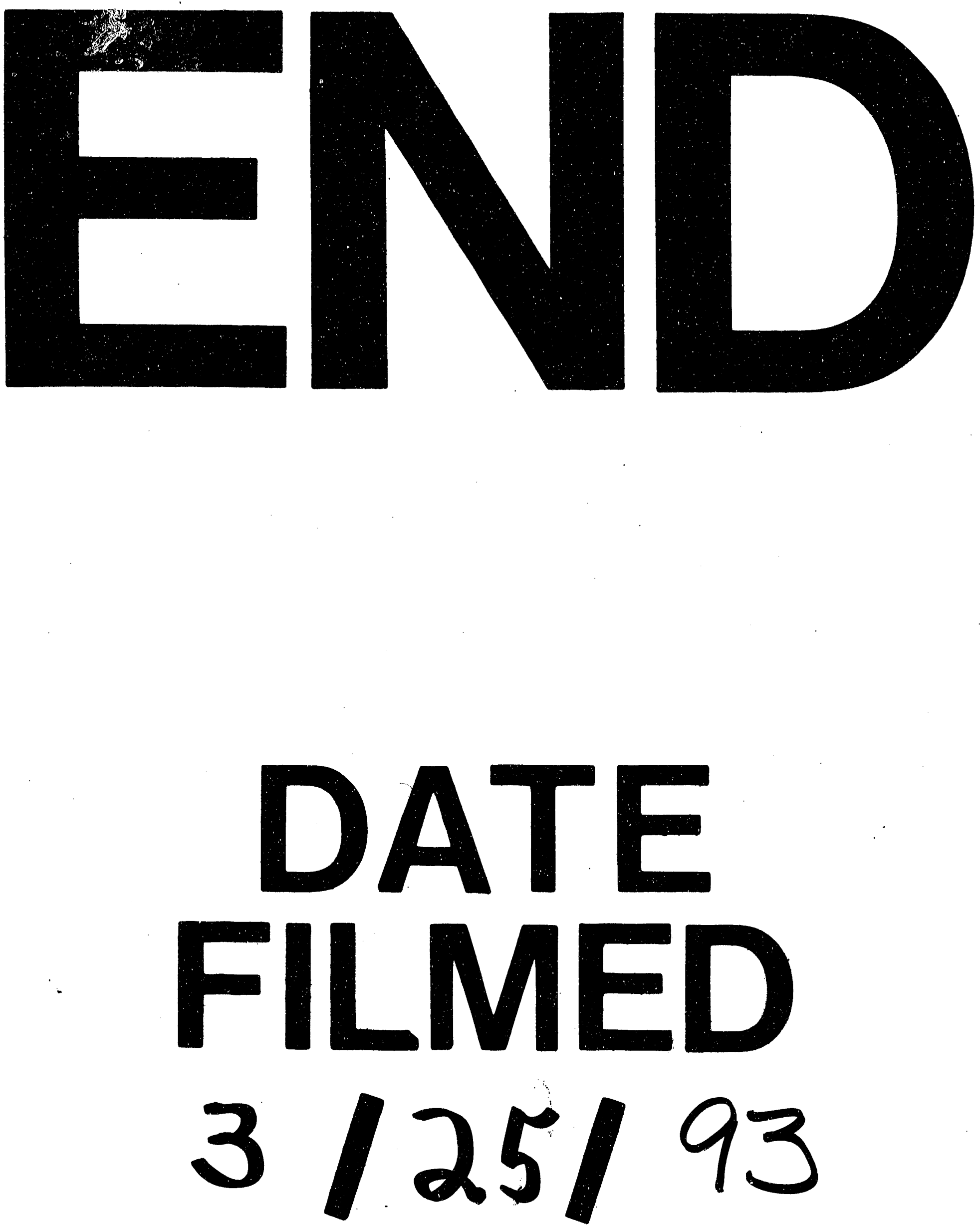
